\title{
Cyclophilin D-dependent oligodendrocyte mitochondrial ion leak contributes to neonatal white matter injury
}

\author{
Zoya Niatsetskaya, ${ }^{1}$ Sergey Sosunov, ${ }^{1}$ Anna Stepanova, ${ }^{1}$ James Goldman, ${ }^{2}$ Alexander Galkin, ${ }^{1}$ Maria Neginskaya, ${ }^{3}$ \\ Evgeny Pavlov, ${ }^{3}$ and Vadim Ten ${ }^{1}$
}

'Department of Pediatrics and 2Department of Pathology and Cell Biology, Vagelos College of Physicians and Surgeons, Columbia University, New York, New York, USA. ${ }^{3}$ Department of Molecular Pathobiology, New York University College of Dentistry, New York, New York, USA.

\begin{abstract}
Postnatal failure of oligodendrocyte maturation has been proposed as a cellular mechanism of diffuse white matter injury (WMI) in premature infants. However, the molecular mechanisms for oligodendrocyte maturational failure remain unclear. In neonatal mice and cultured differentiating oligodendrocytes, sublethal intermittent hypoxic (IH) stress activated cyclophilin D-dependent mitochondrial proton leak and uncoupled mitochondrial respiration, leading to transient bioenergetic stress. This was associated with development of diffuse WMI: poor oligodendrocyte maturation, diffuse axonal hypomyelination, and permanent sensorimotor deficit. In normoxic mice and oligodendrocytes, exposure to a mitochondrial uncoupler recapitulated the phenotype of WMI, supporting the detrimental role of mitochondrial uncoupling in the pathogenesis of WMI. Compared with WT mice, cyclophilin D-knockout littermates did not develop bioenergetic stress in response to IH challenge and fully preserved oligodendrocyte maturation, axonal myelination, and neurofunction. Our study identified the cyclophilin D-dependent mitochondrial proton leak and uncoupling as a potentially novel subcellular mechanism for the maturational failure of oligodendrocytes and offers a potential therapeutic target for prevention of diffuse WMI in premature infants experiencing chronic IH stress.
\end{abstract}

\section{Introduction}

In the United States, each year approximately 60,000 infants are delivered prematurely with very low birth weight (VLBW) of less than $1500 \mathrm{~g}$ (1). In VLBW infants, white matter injury (WMI) accounts for $5 \%-10 \%$ of cerebral palsy and for approximately $50 \%$ of all behavioral and cognitive deficits $(2,3)$. Behavioral problems exist in $34 \%$ of toddlers born at less than 28 weeks of gestation (4). Although the incidence of prematurity remains unchanged, improvement in neonatal survival has been associated with the increasing socioeconomic burden of neurological disability in prematurely born children (2). There are no therapeutic strategies against WMI, and the primary obstacle is our poor understanding of the molecular mechanisms underlying this disease.

WMI has been defined as neuropathological and neuroimage-identifiable extensive tissue loss in the periventricular white matter (WM) associated with neurodevelopmental impairment and cerebral palsy. With advances in neonatal care, the neuropathological spectrum of WMI has shifted away from a massive degeneration of oligodendrocytes (OLs), their precursors, and other cells, known as cystic periventricular leukomalacia (PVL), toward milder forms of WMI, focal gliosis, and/or diffuse hypomyelination (5). Cystic PVL is now diagnosed in only about $5 \%$ of VLBW infants, and noncystic, diffuse WMI has become a dominant lesion $(6,7)$. Mecha-

Conflict of interest: The authors have declared that no conflict of interest exists. Copyright: () 2020, American Society for Clinical Investigation.

Submitted: August 29, 2019; Accepted: July 7, 2020; Published: September 14, 2020

Reference information: J Clin Invest. 2020;130(10):5536-5550.

https://doi.org/10.1172/JCl133082. nistically, WMI is linked to a failure of OL precursor cells (OPCs) and premyelinating OLs (pre-OLs) to reach their myelin-producing state $(6,8)$. PVL is caused by cell-lethal pre- or postnatal insults (hypoxic-ischemic brain injury, intraventricular hemorrhage), but the clinical causes and mechanisms of maturational arrest in surviving OPCs and pre-OLs are not well understood.

Postnatal OL maturation and synthesis of myelin is a highly energy-dependent process (9). In rats, during the initial 3 weeks of life, when the rate of cerebral growth is the greatest relative to any other age (10), mitochondrial oxidative phosphorylating capacity in the brain increases by 11-fold compared with that at birth (11, 12). Energy-dependent biosynthesis in differentiating OLs is very intense, which explains an elevated cerebral metabolic demand during brain development (13). In VLBW-preterm infants, chronic intermittent hypoxemia $(\mathrm{IH})$ is the most common manifestation of cerebral and pulmonary immaturity (14) and has been associated with adverse neurodevelopmental outcomes (15). Previously, we established a mouse model of diffuse WMI without extensive degeneration of OLs or other cells, in which nonlethal, nonischemic IH stress during the initial 2 weeks of life reproduced in mice the phenotype of diffuse WMI in premature infants (16). The mechanisms of diffuse WMI associated with sublethal IH stress are unknown. We hypothesized that IH causes nonlethal mitochondrial bioenergetic dysfunction in actively differentiating OPCs and preOLs, which results in their maturational failure. Using in vivo and in vitro paradigms, we thought to determine whether chronic, nonlethal IH stress in neonatal mice negatively affects mitochondrial function in differentiating OLs, causing bioenergetic deficit-driven OL maturational failure and diffuse WMI. 

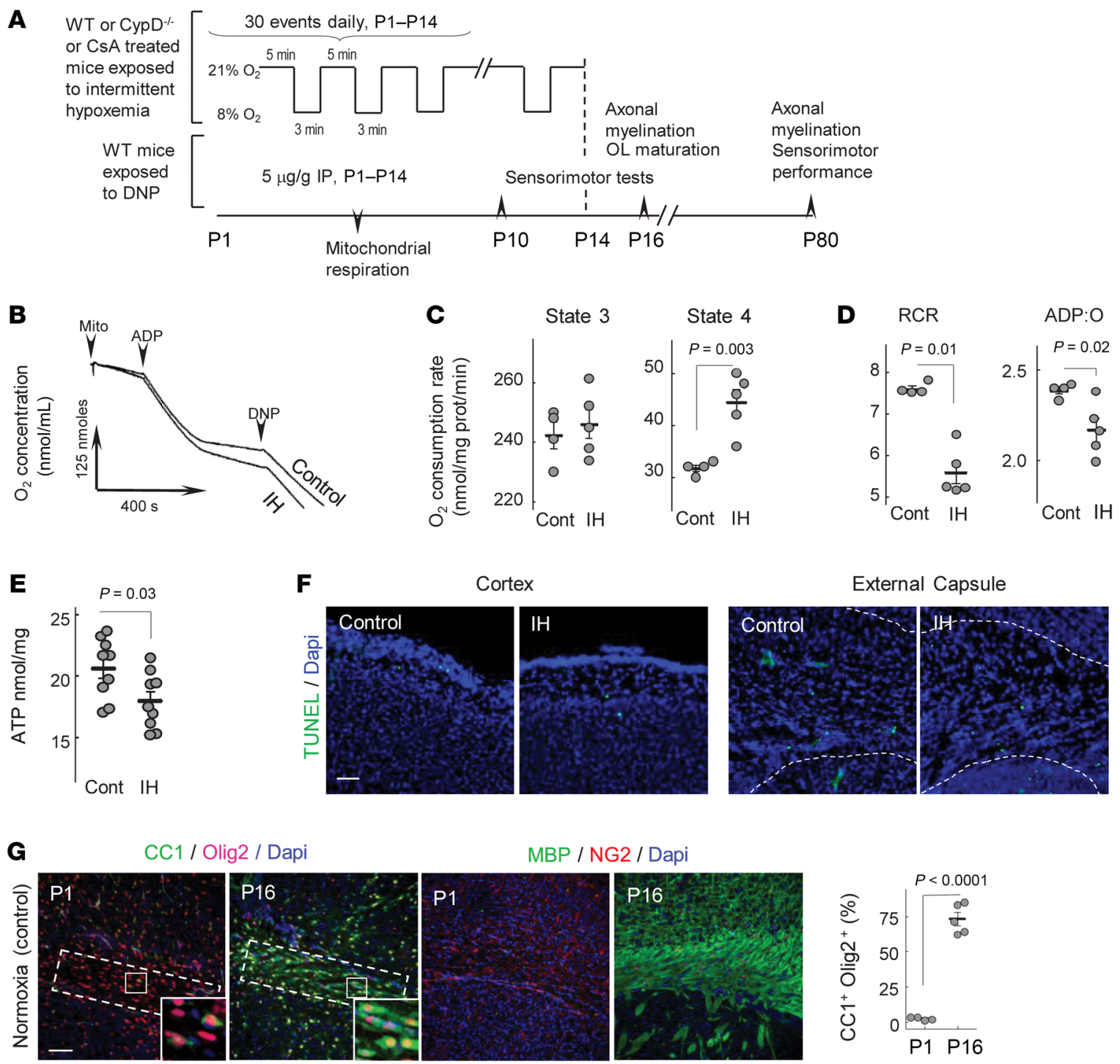

Figure 1. Experimental design and mitochondrial function. (A) IH paradigm with experimental outcomes. (B) Representative tracing of mitochondrial respiration. (C) Mitochondrial states 3 and 4 respiration rates (unpaired $t$ test). (D) Respiratory control ratio (RCR) and ADP/O (nmol of phosphorylated ADP per atom of the $\mathrm{O}_{2}$ ) ratio (Mann-Whitney $U$ test). (E) Cerebral ATP content at the end of 10 IH events (unpaired $t$ test). (F) Immunostaining for TUNEL ${ }^{+}$cells in normoxic and IH P10 mouse brains. Scale bar: $100 \mu \mathrm{m}$. (C) Confocal microscopy of external capsule stained for CC1, Olig2, MBP, NC2, and DAPI with CC1+Olig2+ cell count. Dashed outline is the area selected for assessment of CC1+Olig2+ cell density, expressed as a percentage of total Dapi ${ }^{+}$cells. Scale bar: $100 \mu \mathrm{m}$.

\section{Results}

Sublethal IH stress uncouples respiration in subcortical mitochondria and decreases ATP content. Compared with normoxic controls, subcortical mitochondria isolated from mice exposed to the IH paradigm (Figure 1A) demonstrated significantly accelerated resting (state 4) respiration rates. The phosphorylating (state 3) and dinitrophenol-accelerated (DNP-accelerated) respiration rates remained unaffected (Figure 1, B and $\mathrm{C}$ ). In this assay, a mitochondrial uncoupling agent, DNP, was used to directly probe an activity of the respiratory chain. Intact DNP-uncoupled and state 3 respiration rates suggested unaffected respiratory chain activity in IH. Significantly elevated state 4 respiration (excessive oxygen consumption in the absence of ADP stimuli) defined the state of mitochondrial uncoupling. IH-driven uncoupling of subcortical mitochondria was also evidenced by a significantly poorer respi- ratory control ratio (state 3/state 4 , respiratory control ratio) and decreased ADP/O ratio (the amount of ADP phosphorylated during consumption of 1 atom of the oxygen) compared with controls (Figure 1D). Importantly, compared with controls, the cerebral ATP content at the end of the IH events was significantly decreased (Figure 1E). This bioenergetic stress was not lethal for the cells of interest. The TUNEL assay revealed no excessive cellular death in the cortex (percentage of dead cells $=0.34 \% \pm 0.044 \%$ in $\mathrm{IH}$, control $=0.31 \% \pm 0.17 \%, n=3)$ and the external capsule $(\mathrm{IH}=0.86 \%$ $\pm 0.064 \%$ vs. control $=0.83 \% \pm 0.16 \%, n=3$ ) after 10 days of $\mathrm{IH}$ stress (Figure 1F).

We also confirmed that over the initial 2 weeks of life, control mice demonstrated a developmental spurt in OL maturation and cerebral myelination. Compared with P1 mice, P16 mice displayed a significant increase in the density of mature OLs 
A

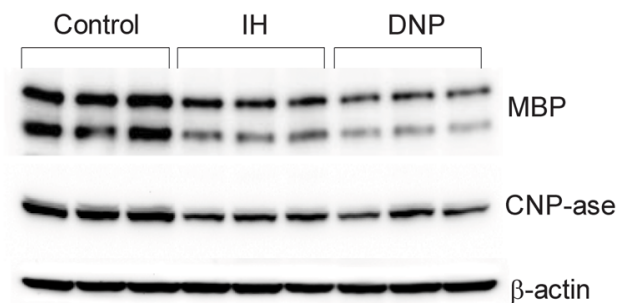

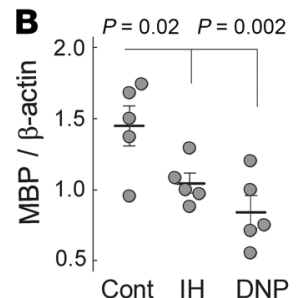

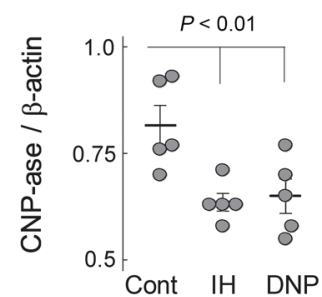

C
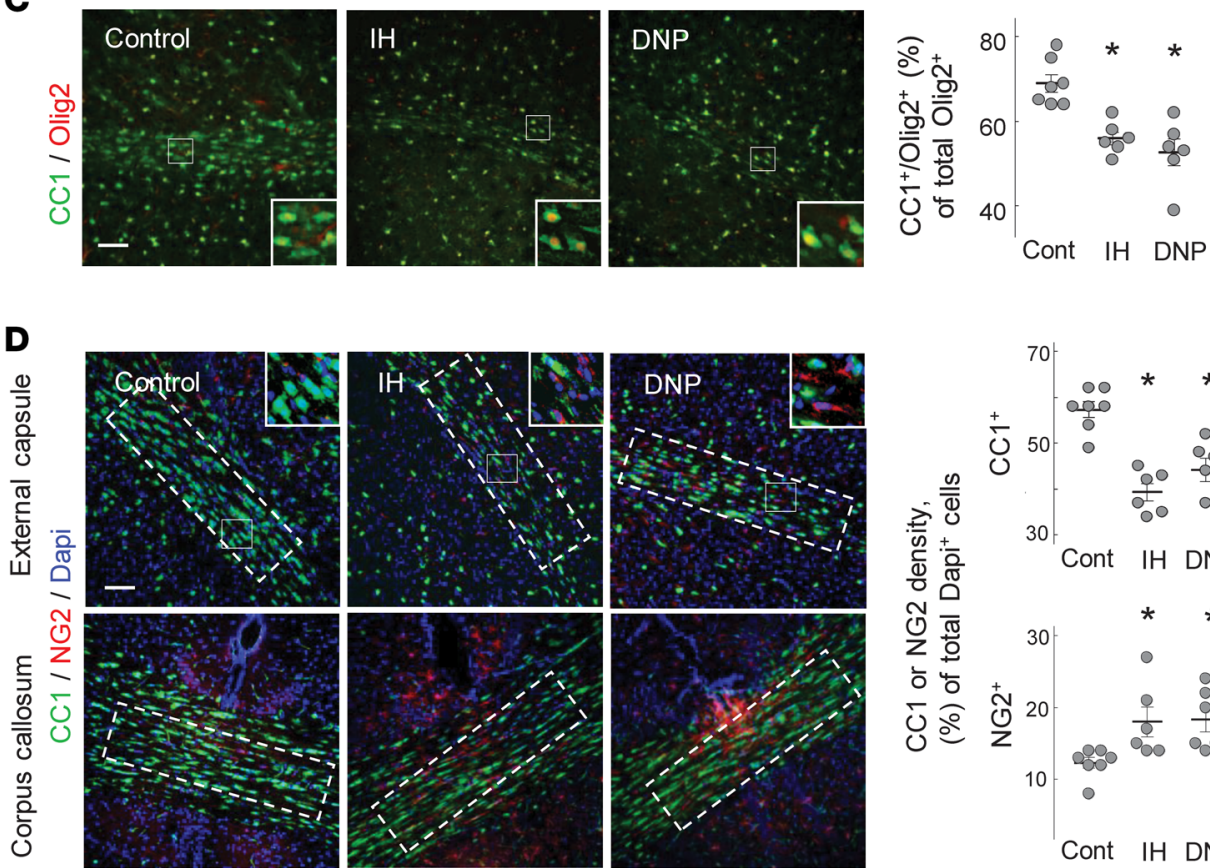

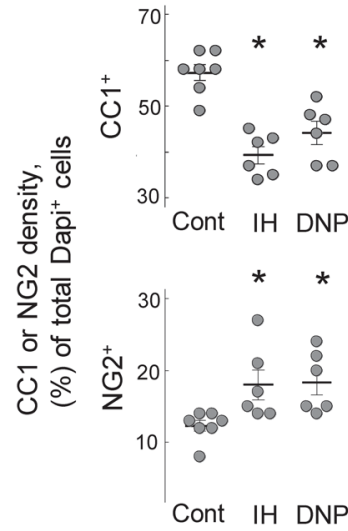

Figure 2. IH or DNP inhibits myelination. (A and $\mathbf{B}$ ) Western blot analysis of the MBP and CNP-ase content in the entire hemisphere of $\mathrm{P} 16$ mice. Groups are indicated. (C) Confocal microscopy of the external capsule and adjacent areas stained for CC1 and Olig2 with analysis of the double-positive cell count in relation to total Olig2 ${ }^{+}$cells. ${ }^{*} P \leq 0.001$; scale bar: $50 \mu \mathrm{m}$. (D) Confocal microscopy of corpus callosum and external capsule in control, IH, and DNP-treated P16 mice. Dashed outlines are the areas selected for assessment of CC1 and NG2 cell density in relation to the total cell count (Dapi+). ${ }^{*} P \leq$ 0.0003 for CC1 cells and $P \leq$ 0.01 for NG2 compared with controls. Scale bar: $100 \mu \mathrm{m}$. One-way ANOVA with Dunnett's post hoc analysis.
(Olig2 ${ }^{+} \mathrm{CC}^{+}$cells) and a dramatic elevation of the cerebral myelin basic protein (MBP) signal (Figure 1G).

IH or pharmacological mitochondrial uncoupling in normoxia inhibits OL maturation. If mice during the initial 2 weeks of life experienced IH stress, MBP and 2'3' cyclic-nucleotide 3-phosphodiesterase (CNP-ase) signals in their brains were significantly attenuated compared with controls (Figure 2, A and B).

The mechanistic significance of mitochondrial uncoupling in the pathogenesis of WMI was addressed by the exposure of normoxic mice to mitochondrial uncoupler DNP. DNP is a classical ionophore and dramatically activates proton flux into the matrix, bypassing ATP synthase (17). Normoxic mice exposed to DNP exhibited significant cerebral hypomyelination compared with vehicle-treated counterparts (Figure 2, C and D). In the corpus callosum and external capsule, IH mice and mice exposed to DNP demonstrated significantly decreased density of mature OLs (Olig2 ${ }^{+} \mathrm{CC}^{+}$cells) compared with normoxic controls (Figure $2, \mathrm{C}$ and $\mathrm{D})$. In contrast, the density of immature OLs $\left(\mathrm{NG}^{+}\right)$in the same areas of the brain was significantly greater in $\mathrm{IH}$ mice and DNP mice compared with controls (Figure 2D). No difference was detected in the total cell counts $\left(\mathrm{Dapi}^{+}\right)$, or the counts of Olig2 ${ }^{+}$ cells between IH mice (Dapi ${ }^{+}, 108.8 \pm 13.1$ /area; Olig2 ${ }^{+}, 97 \pm 17$ / area; $n=6)$, mice treated with DNP $\left(\mathrm{Dapi}^{+}, 115.1 \pm 3.4 /\right.$ area; Olig2 ${ }^{+}$, $92.6 \pm 10.6 /$ area; $n=6$ ), and the control group ( $\mathrm{Dapi}^{+}, 107.8 \pm 14$ / area; Olig2 ${ }^{+}, 99.4 \pm 20.2$ /area; $n=7$, all data mean \pm SD).
The next experiment was performed in vitro using a primary culture of OPCs (NG2 cells). Before differentiation, the majority $(92 \% \pm 4.4 \%$, mean $\pm \mathrm{SD}, n=6)$ of cultured NG2 cells were Olig2 $2^{+}$, confirming the OL precursor lineage (Figure $3 \mathrm{~A})$. When these cells were exposed to IH stress, their differentiation into CNP-ase-expressing or MBP-expressing cells was significantly suppressed compared with their normoxic counterparts (Figure 3, A-D). To demonstrate a contribution of mitochondrial uncoupling to the OL maturational arrest, a different set of differentiating normoxic NG2 cells was intermittently exposed to DNP. The exposure to DNP also arrested the maturation of NG2 cells to a similar extent as the exposure to IH stress (Figure 3, A-D).

Pharmacological mitochondrial uncoupling replicates the WMI phenotype produced by IH. After either the DNP or IH exposure, mice exhibited strikingly similar sensorimotor deficits compared with their control littermates (Figure 4A). Electron microscopy of the corpus callosum and analysis of the g-ratio in these mice revealed significantly poorer axonal myelination and fewer myelinated axons compared with controls (Figure 4, B-D). Because mitochondrial uncoupling may cause excessive heat generation, in a separate cohort of mice we measured the oral temperature in P5 and in the same P7 mice ( $n=7$ per group) hourly, 3 times after the DNP or vehicle injection. No significant difference in the temperature was detected (data not shown). 
A Day 0 of differentiation
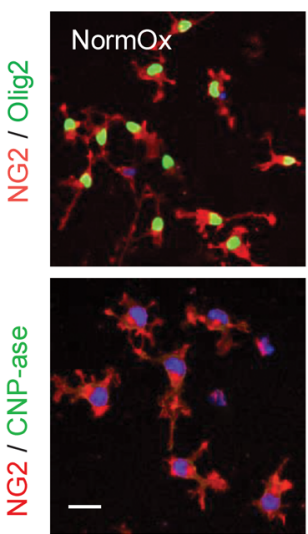

C Day 0 of differentiation

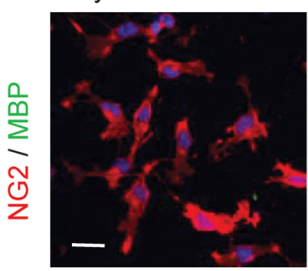

Day 2 of differentiation
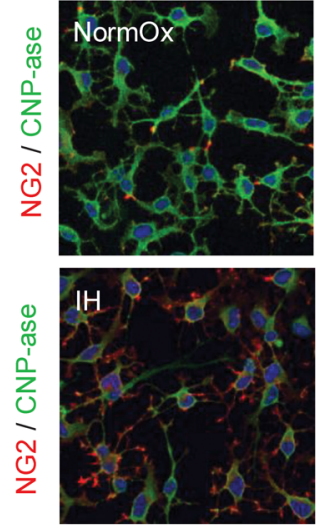

Day 5 of differentiation
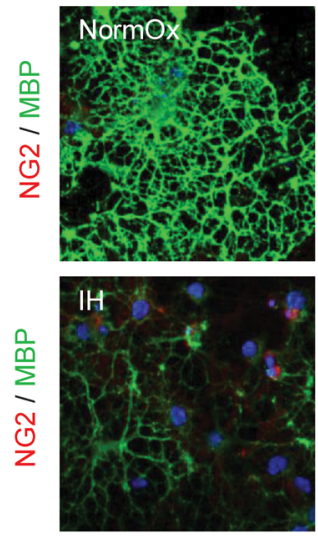
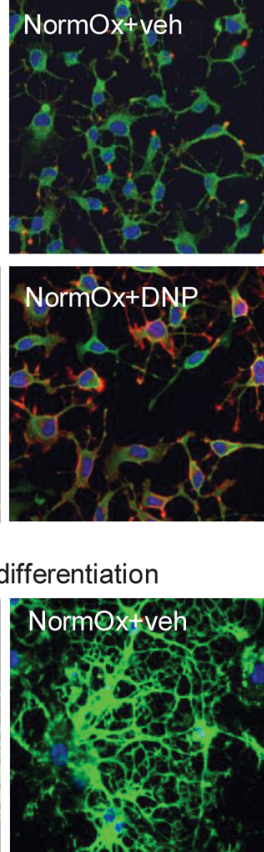

NormOx+DNP

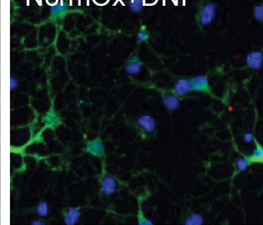

B
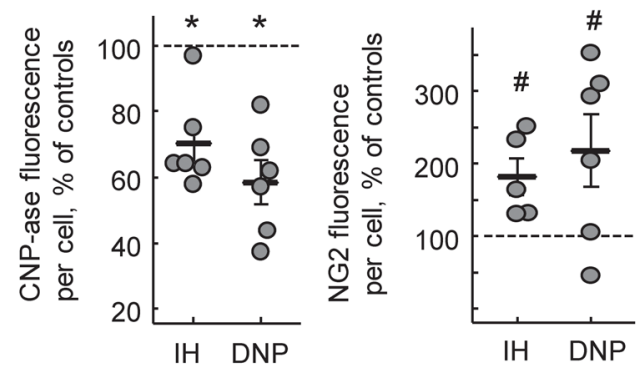

D

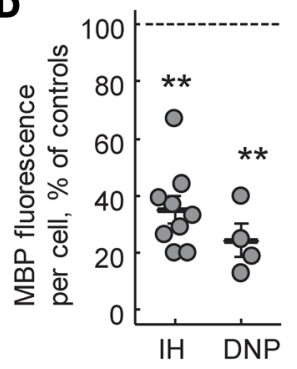

Figure 3. IH or DNP arrests OLs' maturation.IH or DNP arrests OLs' maturation.(A) Confocal microscopy of cultured OPCs stained for NG2 and Olig2 (control for OPC purity) and NG2 and CNP-ase before and after 2 days of differentiation under normoxic, IH, or DNP exposures. Scale bar: $30 \mu \mathrm{m}$. (B) CNPase- and NG2-specific fluorescence per Dapi ${ }^{+}$cell count in the field after 2 days of the OPC differentiation under IH or DNP exposure. Data expressed as the percentage in relation to the fluorescence in the normoxic controls. ${ }^{*} P<0.0005$ (1-way ANOVA, Dunnett's post hoc); ${ }^{*} P<0.01$ (Kruskal-Wallis and Dunn's post hoc tests). (C and D) Images and analysis of MBP fluorescence per cell in the OPCs before and after 5 days of differentiation under normoxic, IH, or DNP exposure. ${ }^{* *} P<0.009$ (1-way ANOVA, Dunnett's post hoc test) compared with controls (NormOx). Blue is DAPI; scale bar: $30 \mu \mathrm{m}$.

IH activates cyclosporine A-sensitive mitochondrial ion leak in differentiating OPCs. So far, we have found that IH stress was associated with bioenergetic dysfunction characterized by mild uncoupling of subcortical mitochondrial respiration. Mitochondrial uncoupling is the function of excessive proton leak across the inner mitochondrial membrane. To probe mitochondrial membrane permeability directly, we used an excised patch-clamp technique and measured the transmembrane electrical conductance in mitochondria isolated from the OPCs differentiating in culture. Compared with normoxia, IH applied during the initial 2 days of differentiation significantly increased the ion permeability of the inner mitochondrial membrane (Figure 5, A and B). We did not detect the ion channel activity, but the ion currents in $\mathrm{IH}$ mitochondria were sensitive to cyclosporine A (CsA, Figure 5A), suggesting that the increased ion leak during IH was linked to the activation of the low-conductance mode of the cyclophilin D-sensitive (CypD-sensitive) permeability transition (18). One of the known CsA actions relates to an inhibition of the mitochondrial enzyme CypD, peptidyl-prolyl cis-trans isomerase, known as the component/activator of the mitochondrial permeability transition pore induced by $\mathrm{Ca}^{2+}$ and other pathological stimuli (19). We subjected differentiating OPC (NG2) cells lack- ing CypD $\left(\mathrm{CypD}^{-/-}\right)$to the same $\mathrm{IH}$ paradigm and compared their mitochondrial membrane permeability with WT IH counterparts. First, we compared the purity of the primary OPC culture of both genotypes (Figure $5 \mathrm{C}$ ). In the WT and $\mathrm{CypD}^{-/-} \mathrm{NG} 2$ cell cultures, Olig2 ${ }^{+}$cells consisted of $92 \% \pm 4.4 \%$ and $93 \% \pm 1.5 \%$ of all $\mathrm{Dapi}^{+}$ cells (mean $\pm \mathrm{SD}, n=6$ and 4 ). The patch-clamp assessment of mitochondria isolated from these cells revealed that, compared with normoxic controls and in contrast to their WT counterparts, $\mathrm{CypD}^{-/-}$ organelles displayed no significant changes in their membrane ion conductance in response to IH (Figure 5B).

The physiological relevance of the mitochondrial ion leak was examined by measuring mitochondrial membrane potential $\left(\Delta \Psi_{\mathrm{m}}\right)$ in differentiating NG2 cells with and without IH stress. IH resulted in partial dissipation of $\Delta \Psi_{\mathrm{m}}$ only in WT cells (Figure 5D). In contrast, CypD ${ }^{-1-}$ differentiating OPCs subjected to IH fully preserved their $\Delta \Psi_{\mathrm{m}}$ compared with their WT IH counterparts (Figure 5, D and E). To highlight the potential mechanism for the activation of mitochondrial permeability transition, we examined intramitochondrial $\mathrm{Ca}^{2+}$ accumulation during $\mathrm{IH}$ stress. Figure $5 \mathrm{~F}$ shows that compared with normoxic controls, isolated subcortical mitochondria accumulated significantly greater $\mathrm{Ca}^{2+}$ at the end of $10 \mathrm{IH}$ episodes. 
A Olfactory discrimination

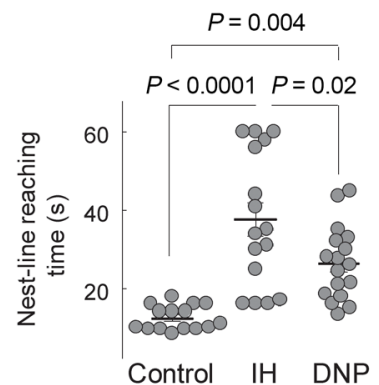

Wire-holding

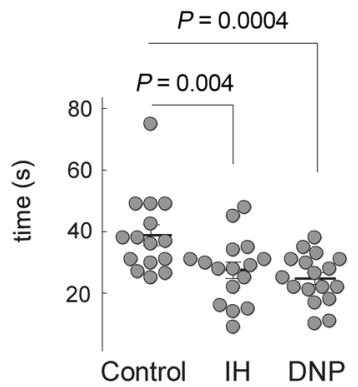

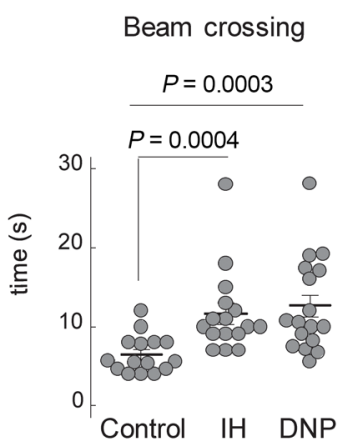

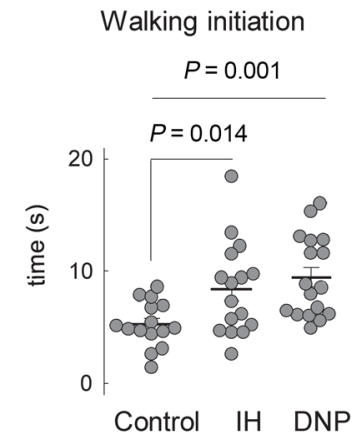

B

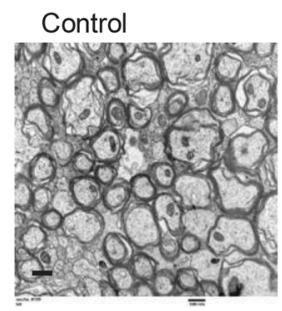

IH

DNP
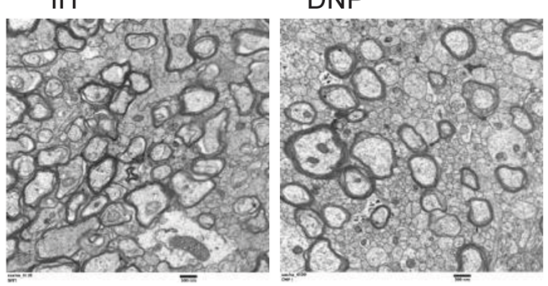

C

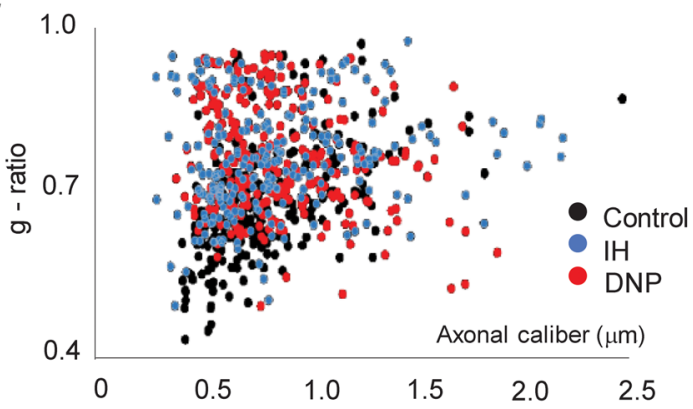

D

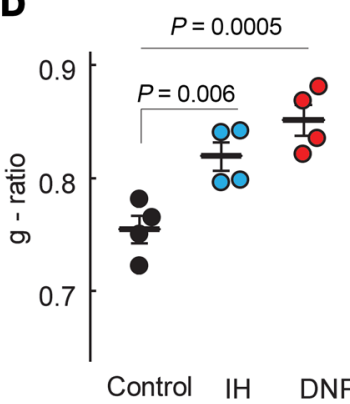

Figure 4. IH or DNP causes sensorimotor deficit. (A) Sensorimotor performance in neonatal mice after IH or DNP exposure. One-way ANOVA, Tukey's post hoc test (olfactory discrimination), 1-way ANOVA, Dunnett's post hoc test (wire holding, walking initiation), and Kruskal-Wallis and Dunn's post hoc tests (beam crossing). (B-D) Electron microscopy of corpus callosum and axonal g-ratio in the same groups of mice. One-way ANOVA, Dunnett's post hoc test. Scale bar: $500 \mathrm{~nm}$ (B).

Genetic deletion of CypD or treatment with CsA protects mice and OPC from IH-driven diffuse WMI and maturational arrest. To explore whether CypD-dependent ion/proton leak contributes to OL maturational failure, we studied the effects of IH stress on OL maturation and axonal myelination in a $\mathrm{CypD}^{-/}$background. $\mathrm{CypD}^{-/-} \mathrm{OPC}$ were remarkably resistant to $\mathrm{IH}$ stress and fully preserved their maturation rates. In contrast, WT OPCs exposed to an identical IH exhibited significantly delayed differentiation compared with their $\mathrm{CypD}^{-/-}$ counterparts (Figure 6, A and B). In vivo, during (P7) and after (P16) $\mathrm{IH}$ exposure, Western blot for cerebral MBP content revealed intact cerebral myelination in $\mathrm{CypD}^{-/} \mathrm{IH}$ mice compared with their WT $\left(\mathrm{CypD}^{+/-}\right.$and $\mathrm{CypD}^{+/+}$) littermates exposed to IH stress (Figure 6, C and $\mathrm{D})$. $\mathrm{CypD}^{-/-} \mathrm{IH}$ mice also exhibited the same density of mature OLs $\left(\mathrm{CC1}^{+}\right.$cells) and OPCs $\left(\mathrm{NG}^{+}\right)$in their corpora callosa and adjacent external capsules as control mice (Figure 7, A-C). In contrast, WT IH mice demonstrated significantly decreased density of $\mathrm{CCl}^{+}$cells and significantly increased density of $\mathrm{NG} 2^{+}$cells compared with controls (Figure 7, A-C). The numbers of total $\mathrm{Dapi}^{+}$cells in both regions did not differ between groups (Figure 7D).

In $\mathrm{CypD}^{-/-}$mice, $\mathrm{IH}$ stress did not alter short-and longterm sensorimotor performance, whereas the WT counterparts demonstrated significant neurofunctional deficits compared with $\mathrm{CypD}^{-1-}$ and control mice (Figure $8, \mathrm{~A}^{-} \mathrm{C}$ ). WT and $\mathrm{CypD}^{-}$ 1- normoxic mice were combined into a single control group, as the genotype did not affect sensorimotor function in controls (wire-holding test, $\mathrm{WT}=161 \pm 27$ seconds, $n=6$ and $\mathrm{CypD}^{-/=}=156$ \pm 13.5 seconds, $n=8$; rotarod test, $\mathrm{WT}=192 \pm 17.5$ seconds, $n=6$ and $\mathrm{CypD}^{-/-}=186 \pm 12$ seconds, $n=8$; all data are mean $\pm \mathrm{SEM}$ ). Importantly, an analysis of cerebral AMPK phosphorylation, a fundamental bioenergetic stress-response system, revealed that only WT mice significantly activated (phosphorylated) AMPK in response to IH stress (Figure 8D). No significant activation of AMPK was found in $\mathrm{CypD}^{-/-} \mathrm{IH}$ mice compared with the genotype-matched controls. Moreover, IH insult did not affect the ATP content of the brain tissue of $\mathrm{CypD}^{-/-}$mice (Figure 8D, also compare with Figure 1E, for the WT). Importantly, the extent of systemic oxygenation $\left(\mathrm{SpO}_{2}\right)$ measured in $\mathrm{P} 10$ mice during $\mathrm{IH}$ events did not differ between genotypes. The mean \pm SD values of minimal $\mathrm{SpO}_{2}$ during a single hypoxic event were $63.8 \% \pm 5.1 \%$ in WT mice and $62.4 \% \pm 3.7 \%$ in their $\mathrm{CypD}^{-/-}$counterparts $(n=$ 5). In adult $\mathrm{CypD}^{-/-}$mice exposed to IH stress in their neonatal age, electron microscopy of corpora callosa revealed significantly better-preserved axonal myelination and g-ratio compared with their WT IH littermates (Figure 8, E and F). 
A Normoxia

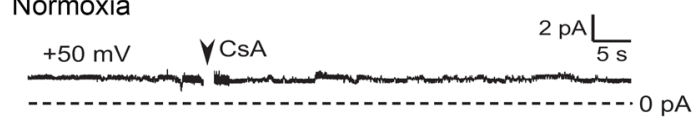

$\mathrm{IH}$

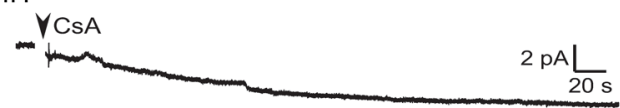

$+30 \mathrm{mV}$
B

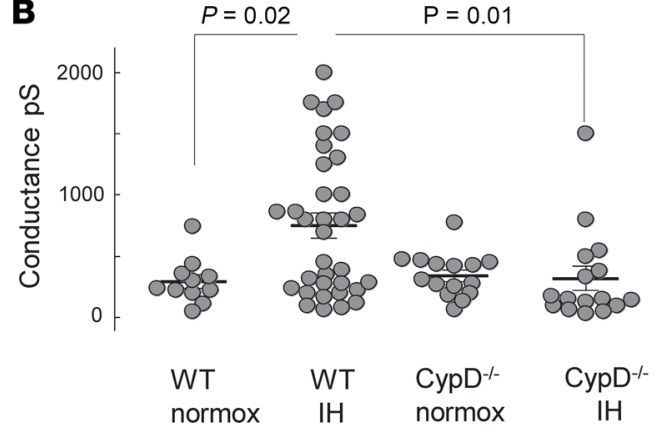

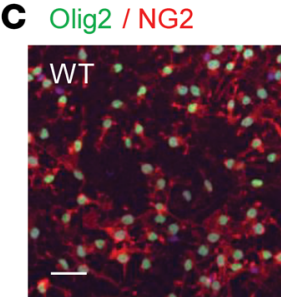

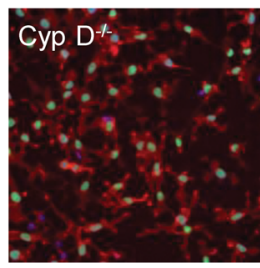

Dapi
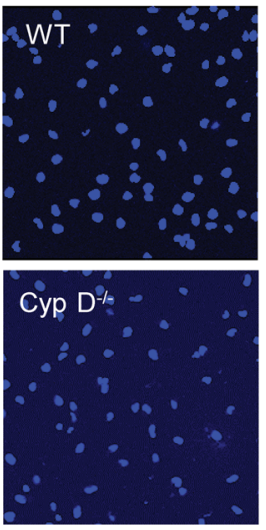

D

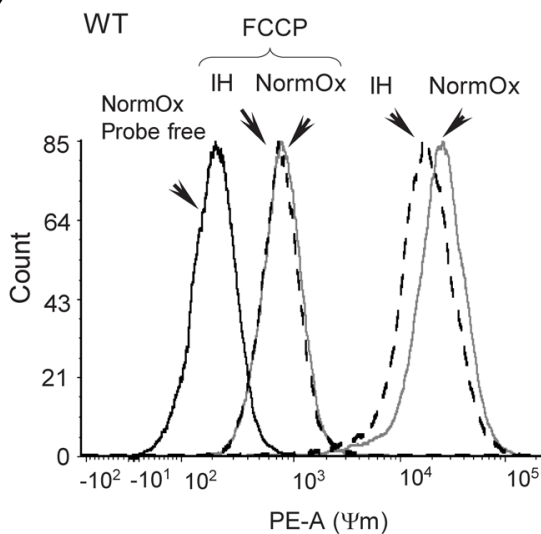

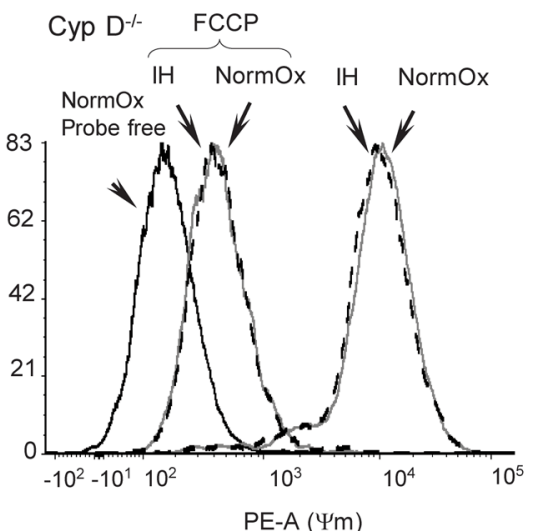

E

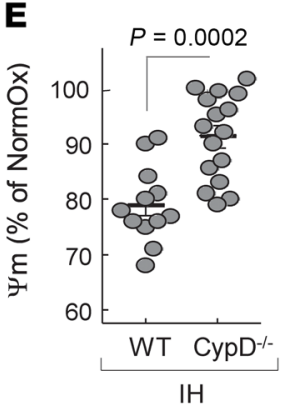

$\mathbf{F}$

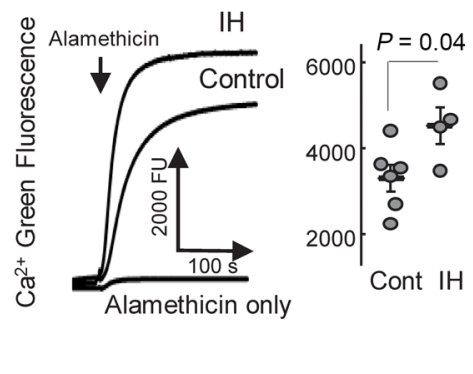

Figure 5. IH activates mitochondrial ion leak. (A) Ion leak-driven conductance in the mitoplasts from WT OPCs after 2 days of differentiation under normoxia or IH. CsA, $2 \mu \mathrm{M}$. pA, picoAmpere. (B) Electrical conductance in mitoplasts isolated from differentiating WT or CypD ${ }^{-1-}$ OPCs after 2 days of maturation under normoxia or IH. Two-way ANOVA with Tukey's post hoc test. (C) Representative images of OPC primary culture stained for Olig2, NG2, and DAPI showing approximately $92 \%$ of Olig2 ${ }^{+}$cells (values are in the text). Scale bar: $50 \mu \mathrm{m}$. (D) Representative tracing of tetramethylrhodamine, ethyl ester (TMRE) fluorescence $\left(\Delta \Psi_{\mathrm{m}}\right)$ distribution in WT and $\mathrm{CypD}^{-1-}$ OPCs after 2 days of differentiation under normoxia (solid line) or IH (dashed line). Left peak is the probefree control; FCCP-pointing peak is the fluorescence distribution with collapsed $\Delta \Psi_{\mathrm{m}}$. PE-A, phycoerythrin $\mathrm{A}$. (E) $\Delta \Psi_{\mathrm{m}}$ as a function of TMRE fluorescence in WT or $\mathrm{CypD}^{-/-}$OPCs after 2 days of differentiation under IH stress. Unpaired $t$ test. (F) $\mathrm{Ca}^{2+}$ fluorescence after alamethicin-induced "piercing" of membranes in subcortical mitochondria isolated from controls and mice after $10 \mathrm{IH}$ episodes with statistical analysis of the $\mathrm{Ca}^{2+}$ fluorescence values at 200 seconds of recording. Unpaired $t$ test.

Comparative analysis of the extent of the cellular death associated with $\mathrm{IH}$ stress in neonatal (P5) WT and $\mathrm{CypD}^{-/-}$mice revealed that compared with controls, IH stress did not increase cellular death in WT or CypD ${ }^{-/-}$mice (Figure 9A). Of note, caspase-3-positive cells were mostly found in the neuronal layers I and II, and none of the dead cells were positive for Olig2 (Figure 9A).

To highlight a potential translational significance of neuroprotection in animals lacking CypD, WT mice were treated with CsA during IH stress. Compared with the vehicle-treated littermates, CsA-treated mice exhibited significantly greater preserved OL maturation in the external capsule (Figure 9, B and C). The tolerance to IH in these mice was also evidenced by sig- nificantly better performance on an olfactory discrimination test and a strong tendency $(P=0.07)$ for improved performance in the wire-holding challenge compared with the vehicle-treated counterparts (Figure 9D).

\section{Discussion}

At birth, the brain of VLBW premature infants is poorly myelinated and contains mostly premyelinating OLs $(20,21)$. In newborn mice, the cerebral WM is also nearly myelin free and contains only a few mature OLs. By 2 weeks of life, normoxic mice demonstrated a dramatic increase in cerebral myelination and the density of mature OLs (Figure 1G). These data support the selected age 
A
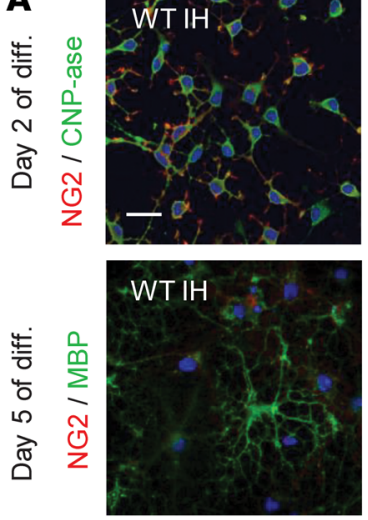

C

P7
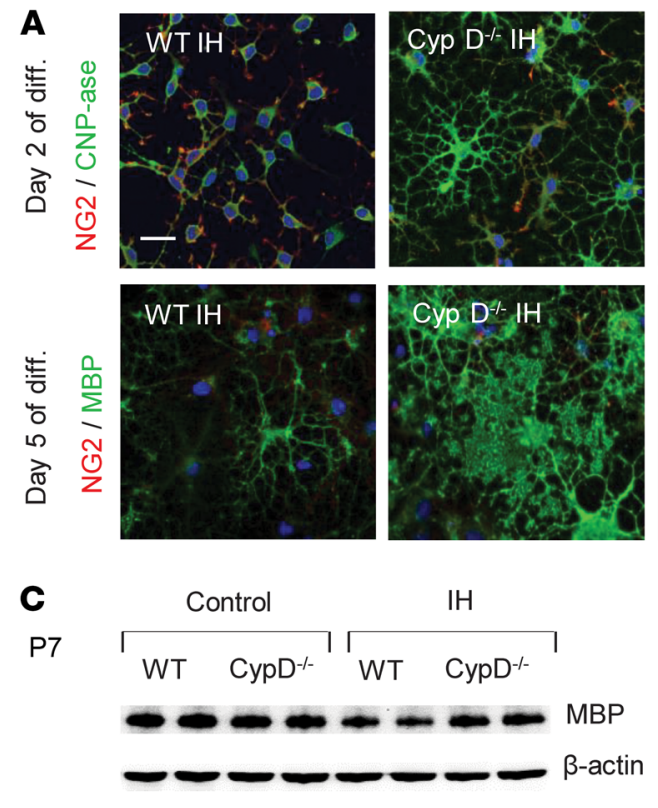

$\mathrm{IH}$
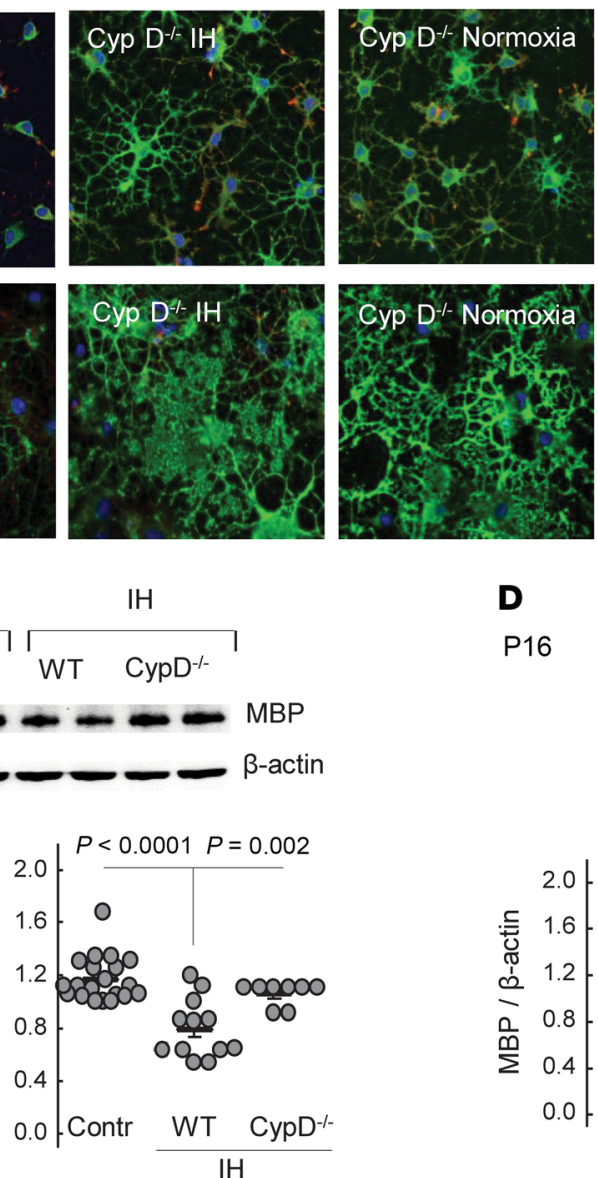

B

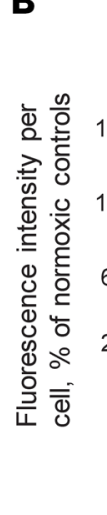

MBP,

on day 5
$P<0.0001$

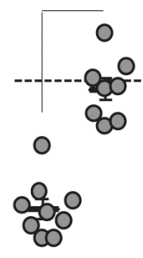

WT CypD--

$\mathrm{IH}$
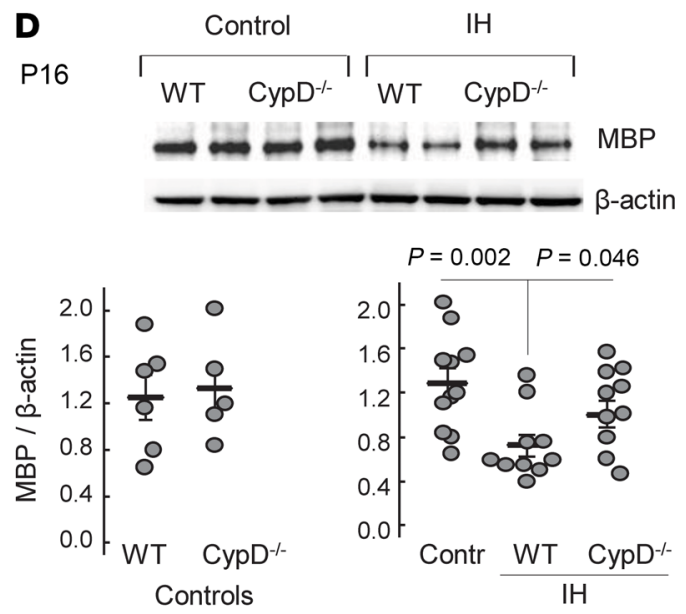

Figure 6. CypD mediates IH-induced arrest of OLs' maturation. (A) Confocal microscopy of WT and CypD ${ }^{-/-}$OPCs stained for NG2 and CNP-ase or NG2 and MBP after 2 or 5 days of differentiation under IH or normoxic conditions. Scale bar: $50 \mu \mathrm{m}$. (B) Analysis of the CNP-ase fluorescence per cell after 2 days of maturation and the MBP fluorescence per cell after 5 days of maturation under IH conditions. Data expressed as the percentage in relation to the normoxic genotype-matched controls (100\%): CNP-ase (WT $\left.n=6, \mathrm{CypD}^{-/-} n=4\right)$ and for MBP (WT $n=9$; CypD $\left.D^{-/-} n=4\right)$. Unpaired $t$ test. (C and D) Cerebral MBP immunoblot images and content in neonatal WT and $\mathrm{CypD}^{-/-}$mice after 7 days of $\mathrm{IH}$ exposure (P7) and upon completion (P16) of IH exposure. Because of no difference between WT and CypD ${ }^{-/-}$controls in the MBP content, both genotypes were combined into a single control group. One-way ANOVA, Dunnett's post hoc test.

for the experimental alteration of WM development in mice, as the most intense OL maturation was observed during the initial week of life $(22,23)$. A maturational failure of OLs to reach their myelin-producing state is viewed as a central cellular mechanism of diffuse WMI in premature neonates $(6,8)$. However, the mechanisms for OLs' maturational failure, especially those not related to cellular degeneration, remain cryptic. We found that functionally intact mitochondria are important for OPC differentiation and myelin synthesis during the postnatal spurt in WM development. This implies that postnatal stress affecting mitochondrial energy production inhibits OL maturation. Using the sublethal IH paradigm, we report that mild IH stress, closely mimicking chronic, nonischemic IH events in premature infants (16), suppressed OL maturation in mice and cultured differentiating OPCs. The OL maturational failure driven by this IH stress was associated with cell-nonlethal bioenergetic stress, evidenced by mitochondrial uncoupling, decreased cerebral ATP content, and elevated AMPK phosphorylation. In turn, OL maturational failure was coupled with diffuse axonal hypomyelination and permanent sensorimotor neurological deficits. In vitro, IH stress also significantly suppressed the maturation of OPCs and partially dissipated $\Delta \Psi_{\mathrm{m}}$, confirming a proton leak-driven mitochondrial uncoupling. Furthermore, direct patch-clamp probing of mitochondria isolated from differentiating OL precursors revealed that $\mathrm{IH}$ activated CsA-sensitive and CypD-dependent mitochondrial ion/proton leakage. Thus, IH-induced mitochondrial uncoupling could be one of the molecular mechanisms driving OL maturational failure. Indeed, exposure of normoxic mice to a mitochondrial uncoupler, DNP $(17,24,25)$, fully recapitulated the phenotype of diffuse WMI produced by IH stress. By arresting the differentiation of cultured OPCs with exposure to DNP, we addressed a specificity of mitochondrial uncoupling to OL maturational failure. These findings are consonant with reports on arrested OPC differentiation after exposure of NG2 cells to another mitochondrial uncoupler, carbonyl cyanide 4-(trifluoromethoxy) phenylhydrazone (FCCP) (26).

In our cell-nonlethal $\mathrm{IH}$ paradigm, mitochondrial dysfunction was limited to uncoupling. Others have reported a profound inhibition of the respiratory chain in the brain mitochondria by continuous IH stress combined with hypercapnia (4 minutes every 6 minutes, 24 hours a day, for 2 weeks), which resulted in extensive neuronal degeneration (27). More severe compared with our paradigm, IH exposure $\left(5.7 \%-8 \% \mathrm{O}_{2}\right.$ for 2 minutes, 30 episodes per hour, for 6 
A
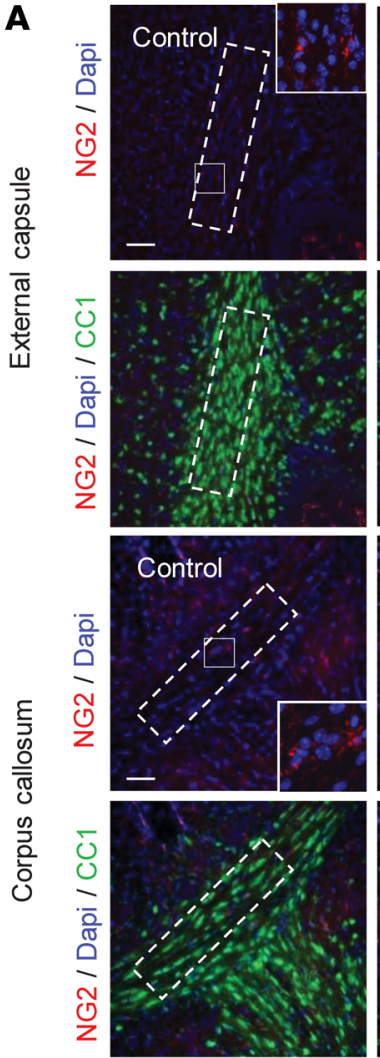
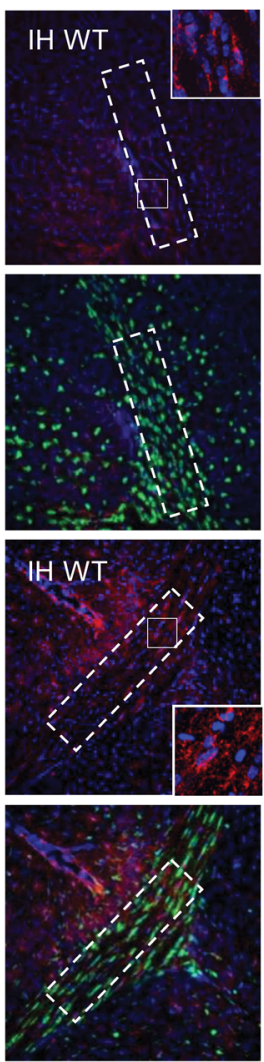
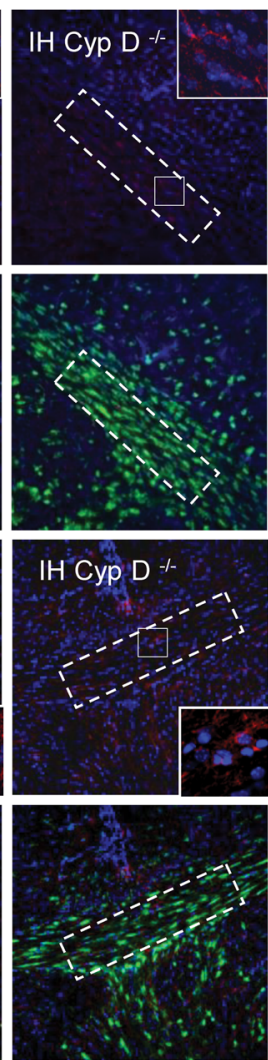

B

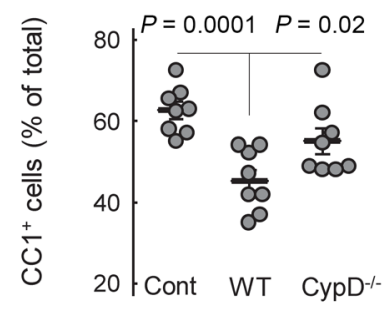

C

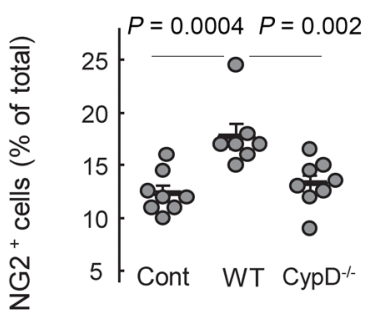

D

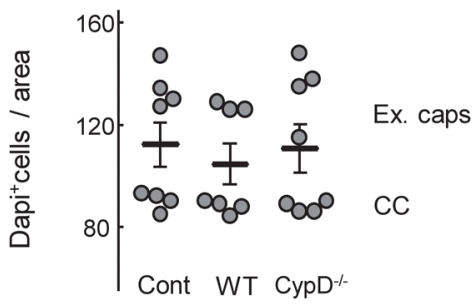

Figure 7. CypD gene KO preserves OLs' maturation during IH. (A) Representative images of external capsule and corpus callosum with adjacent tissues obtained upon completion of IH stress and stained for NG2, CC1, and DAPI. Dashed outline represents the frame selected for assessment of cellular count. Scale bar: $100 \mu \mathrm{m}$. (B and C) Cellular count expressed as the percentage of total Dapi+ cells. One-way ANOVA, Dunnett's post hoc test. (D) Dapi+ cell count in the outlined areas of corpora callosa and external capsules.

hours per day for 8 days, or $1.5 \% \mathrm{O}_{2}$ for 15 minutes, 7 events a day, 3 days) resulted in diffuse cerebral hypomyelination (28) and extensive death of OL precursors and neurons (29). It is important that diffuse axonal hypomyelination in our model occurred in the absence of excessive cellular degeneration in the brain (ref. 16, Figure 1F, and Figure 9A). These data (a) exclude the mechanisms associated with extensive cellular loss in this model of WMI and (b) dissect a potentially novel mechanism: nonlethal mitochondrial dysfunction. We propose that in the absence or minimal death of OLs and other cells, nonlethal mitochondrial dysfunction (excessive proton leak) in differentiating OPCs contributes to developmental myelination failure in premature infants. The potential clinical significance of our work could be underscored by a prognostic uncertainty on whether sublethal chronic IH stress causes WMI and neurodevelopmental impairment in premature human neonates $(14,30)$. Figure $10 \mathrm{~A}$ depicts the potential clinical relevance of animal research in the field. Of note, the reported $(28,29)$ models mimic prolonged and severe $\mathrm{IH}$ events (animals were exposed to hypoxia for up to $40 \%$ of the daily time), addressing the mechanisms of poor neurological outcomes proposed to be associated with severe IH stress (30). Our paradigm mimics more common mild IH stress (mice were hypoxemic for $4.67 \%$ of the daily time) (16) of unknown clinical outcomes (30). In that respect, our study not only offers the mechanism for OPC maturational failure and diffuse WMI but also suggests that neonatal cell-nonlethal IH stress permanently arrests cerebral myelination.
Biophysically, mitochondrial uncoupling implies the presence of proton conductance/leak across the inner mitochondrial membrane. This accelerates mitochondrial respiration without stimulation of ATP production. Compared with normoxic cells, mitochondria from IH-exposed differentiating OPCs exhibited a significantly greater CsA-sensitive ion leak, evidenced by elevated transmembrane electrical conductance. Although the molecular identity of this event is unknown, a sensitivity to CsA suggests that this is a CypD-dependent process. Indeed, compared with the WT counterparts, mitochondria from the differentiating CypD ${ }^{-/-}$ OPCs exhibited a complete tolerance to IH stress, evidenced by preserved transmembrane electrical conductance, intact $\Delta \Psi_{m}$, and normal OL maturation in the IH environment. Because WT OPCs in response to the identical IH stress exhibited a dissipation of the $\Delta \Psi_{\mathrm{m}}$ along with activation of mitochondrial ion leak and arrested differentiation, CypD-dependent mitochondrial membrane permeabilization could be viewed as an intrinsic molecular mechanism of OPC differentiation failure driven by IH. Indeed, in vivo, mature $\mathrm{CypD}^{-/-}$mice subjected to $\mathrm{IH}$ stress in the neonatal age fully preserved their axonal myelination and sensorimotor performance, whereas WT IH littermates exhibited hypomyelination and permanent neurological deficits. This is the first report demonstrating a mechanistic role of CypD-dependent mitochondrial proton leak in actively differentiating OPCs in the pathogenesis of diffuse WMI induced by nonlethal IH stress. 
A

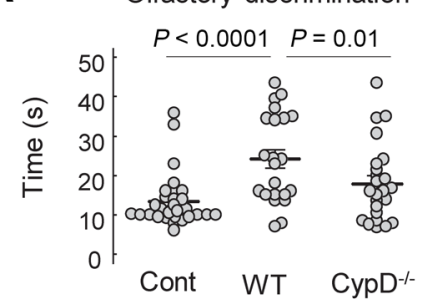

Wire holding

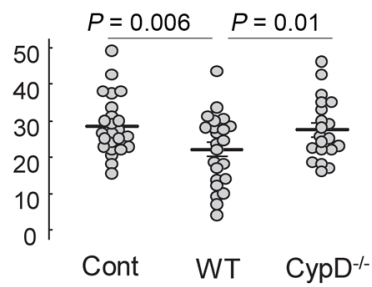

Walking initiation

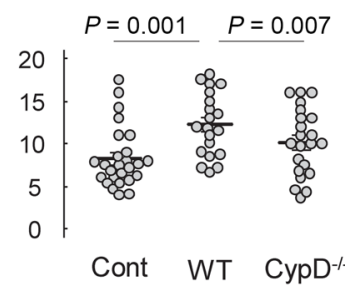

Beam crossing

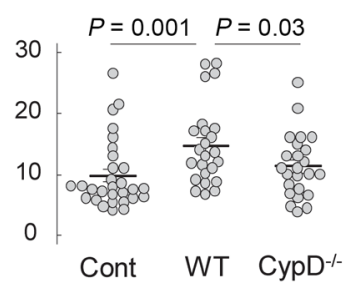

B

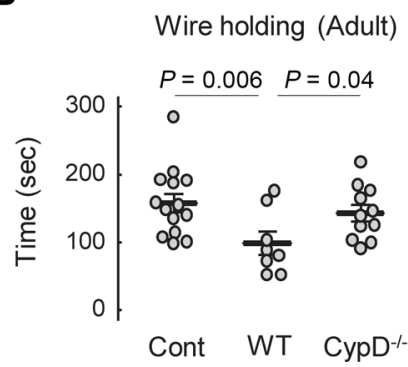

\section{C}

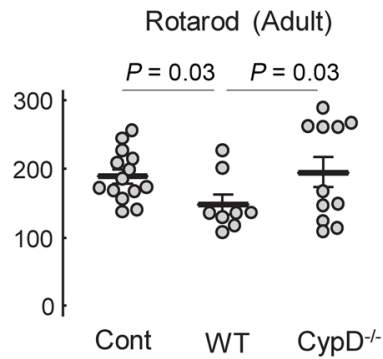

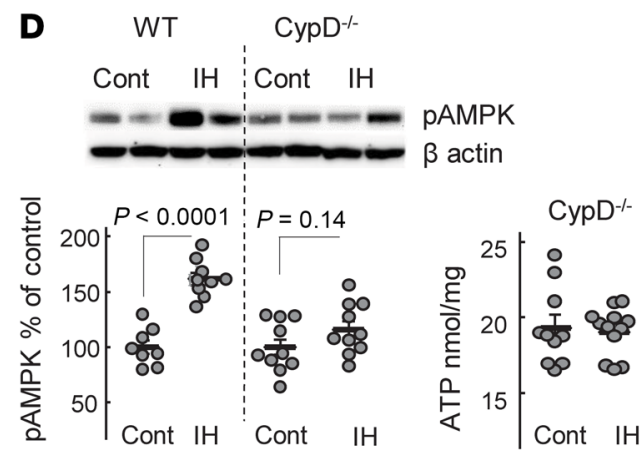

E

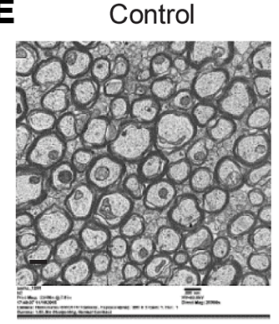

IH CypD ${ }^{+/-}$

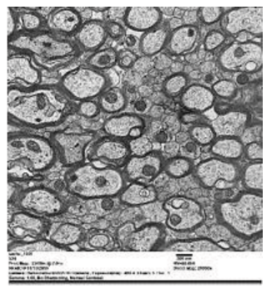

IH CypD ${ }^{-1-}$

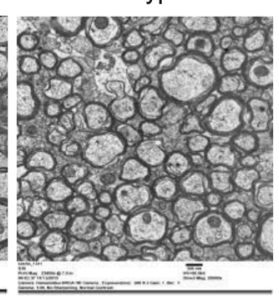

$\mathbf{F}$

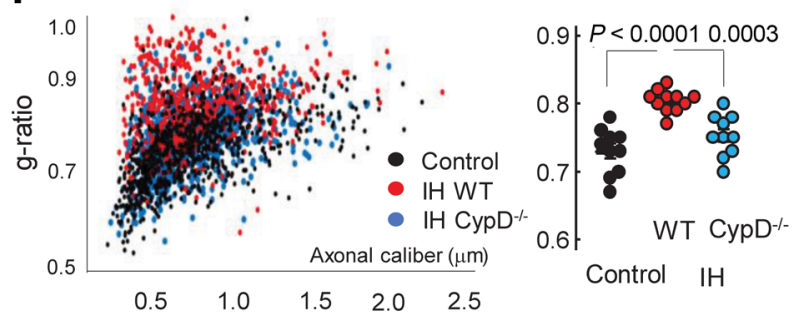

Figure 8. CypD-KO mice are protected against WMI. (A) Sensorimotor performance of WT and CypD-/- mice after IH exposure in comparison with normoxic controls (combined genotypes). Kruskal-Wallis and Dunn's post hoc tests. (B and C) Sensorimotor performance of adult control, WT, and CypD ${ }^{-/-}$mice exposed to IH stress in their neonatal age. One-way ANOVA, Dunnett's post hoc test (B) and Kruskal-Wallis and Dunn's post hoc tests (C). (D) Western blot analysis of phosphorylated (activated) AMPK in WT and CypD ${ }^{-/-}$mice and cerebral ATP content in CypD $D^{-/-}$control and IH mice. Unpaired $t$ test. (E) Representative images of electron microscopy of corpus callosum in adult mice of different genotype exposed to neonatal IH stress compared with control (WT). Scale bar: $500 \mathrm{~nm}$. (F) Analysis of axonal g-ratio estimated using electron microscopy images of corpus callosum in adult mice. One-way ANOVA, Dunnett's post hoc test.

The insight into the upstream cause of excessive mitochondrial leakage revealed a significant $\mathrm{Ca}^{2+}$ accumulation in $\mathrm{IH}$ mitochondria. Given the nonlethal nature of the IH stress, the extent of mitochondrial $\mathrm{Ca}^{2+}$ accumulation is likely to be distinct from the mitochondrial $\mathrm{Ca}^{2+}$ accumulation and derangement activating permanent mitochondrial permeability transition pore and organelle swelling, in addition to cell death in ischemia/reperfusion and other lethal insults $(19,31)$. In physiological conditions, mitochondrial membranes may transiently become ion permeable by activating a low-conductance permeability for $\mathrm{Ca}^{2+}$ efflux to mitigate $\mathrm{Ca}^{2+}$ overload (32). This causes temporary mitochondrial depolarization, which in the healthy state is asynchronous among the organelle population, leaving cellular bioenergetics unaffected (33). IH stress, however, is systemic and simultaneously affects all cells; therefore, a low-conductance mitochondrial permeability is expected to be synchronized with IH events. These IH-driven mitochondrial depolarization episodes will negatively affect the organelles' capacity to produce ATP. We propose that the end biological effect of transient CypD-dependent proton leak is a bioenergetic insufficiency to support the proper development of OL lineage cells and axonal myelination. Indeed, in contrast to the WT mice, CypD ${ }^{-/-}$mice exhibited virtually intact cerebral ATP content and the absence of the AMPK activation in response to IH. The AMPK system activates by elevation of the AMP/ATP ratio and represents a fundamental adaptive response to a bioenergetic deficit, shifting cellular metabolism toward a catabolic state (34). The absence of AMPK activation in $\mathrm{CypD}^{-/-}$ IH mice was consistent with the preserved cerebral ATP content and strongly supports a mechanistic role of CypD-dependent mitochondrial dysfunction in the bioenergetic failure driving OL developmental arrest during $\mathrm{IH}$.

In immature rats, the formation and compaction of the myelin sheath is energy dependent because pharmacological inhibition of mitochondrial respiration significantly reduces developmental myelination (35). In mature rats, the maintenance of the myelin sheath was nearly ATP independent (35). Fünfschilling et al., using a conditional COX10 (a critical component of complex IV) knockout in mature mice, demonstrated that mature OLs are glycolytic and maintain normal axonal myelination, even with blocked oxidative phosphorylation (36). Under optimal in vitro 
A

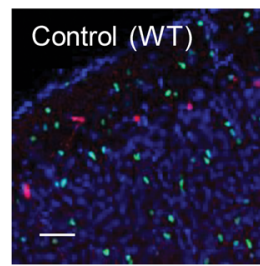

B

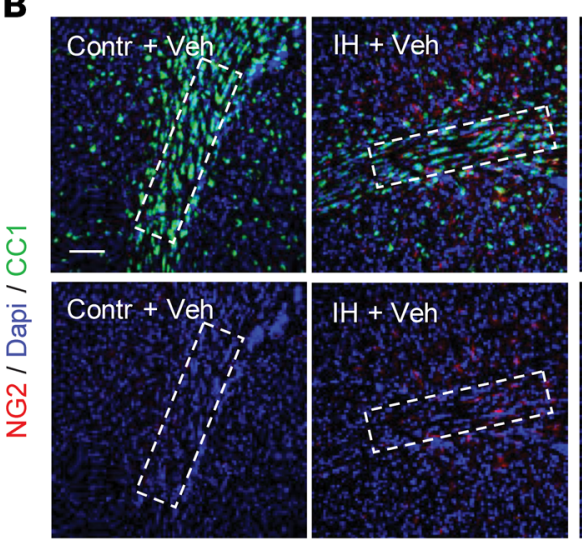

Caspase 3 / Dapi / Olig2
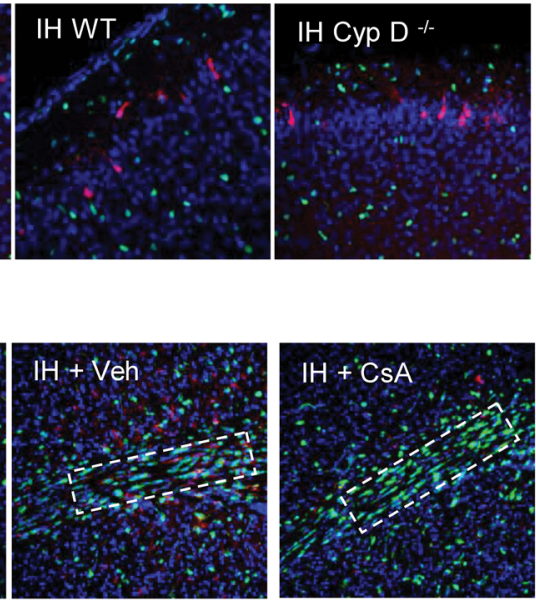

$\mathrm{IH}+\mathrm{CsA}$

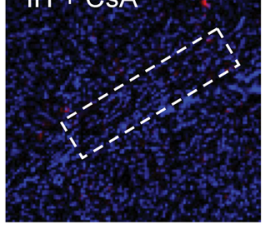

Figure 9. CsA attenuates sensorimotor deficit and preserves OL maturation. (A) Images of the cortical areas stained for cleaved caspase-3, Olig2, and DAPI with analysis of caspase $-3^{+}$cell count relative to all Dapi+ cells in the field. Only fields containing no less than 2 caspase- $3^{+}$cells were analyzed (see Methods). Scale bar: $100 \mu \mathrm{m}$. (B) Confocal microscopy images of external capsules stained for NG2, DAPI, and CC1 in P16 mice exposed to IH and CsA or vehicle. Outlined areas used for CC1 and NG2 count analysis are shown. Scale bar: $100 \mu \mathrm{m}$. (C) CC1 and NG2 cell count in different groups. (D) Sensorimotor performance after IH exposure in the same groups. All analysis done with 1-way ANOVA, Dunnett's post hoc test.

conditions, mature OLs preferentially use glycolysis for their metabolic needs, whereas OPCs mainly use oxidative phosphorylation to produce energy (37). Differentiating OLs were highly sensitive to inhibition of their respiratory chain and arrested their maturation in response to sublethal suppression of mitochondrial complex I (38) or complex IV (39). Protection of complex I with hyperforin preserved mitochondrial ADP-phosphorylating activity and increased expression of OL maturational markers (40).

Mitochondrial uncoupling reported here may not be limited to only OLs' bioenergetic failure to support their maturation. In addition to OLs, subcortical mitochondria studied here also originated from neurons and other glial cells, the proportion of which, depending upon the subcortical region, exceeds or is comparable to that of OLs (41). Mitochondrial uncoupling in other cells (i.e., neurons) may also contribute to the mechanisms of OL maturational failure and hypomyelination. For example, nonsynaptic, nonvesicular release of ATP from axons through volume-activated anion channels has been implicated in the adenosine-driven mechanism of axonal inhibition of OPC proliferation and stimulation of their maturation and axonal myelination $(42,43)$. Given that OPCs fully express adenosine and metabotropic ATP receptors, it is conceivable that neuronal IH-driven mitochondrial uncoupling causing deficient ATP production alters this axonal purinergic signaling for OPC devel-

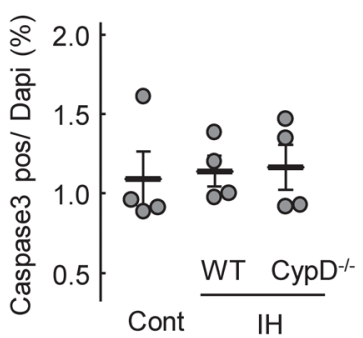

C
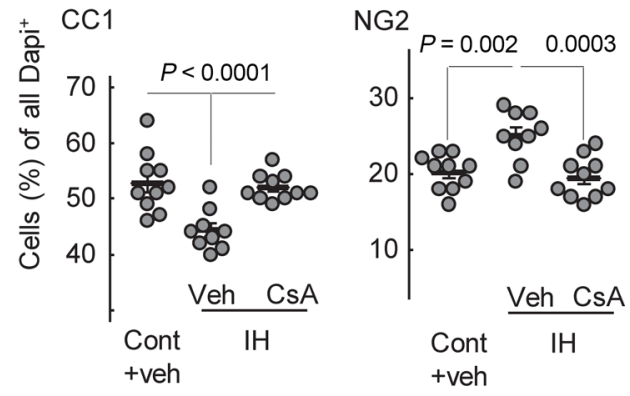

D
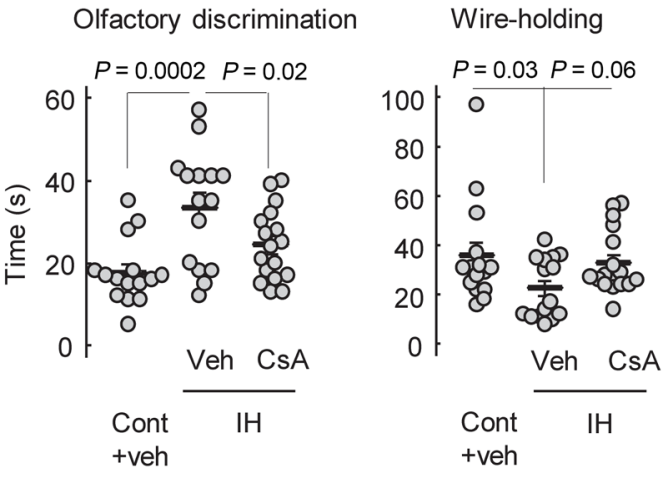

opment. In paving an avenue for testing this hypothesis in the future, we have found that the mitochondria isolated from the cortex of IH mice, where two-thirds of the total cell population are neurons (40), exhibited the CsA-sensitive low-conductance ion leak very similar to that of organelles from differentiating IH-OPCs (Figure 10B). These data suggest that CsA-sensitive mitochondrial membrane leakage in response to nonlethal $\mathrm{IH}$ was not restricted to OL lineage cells. Although the role of nonlethal mitochondrial dysfunction in neurons and other cells in the pathogenesis of diffuse WMI requires further studies, a mechanistic contribution of OPC-specific mitochondrial dysfunction to this disease is reported here.

Next, it is known that even mild mitochondrial uncoupling significantly reduces the generation of ROS in mitochondria (44), especially during succinate oxidation $(45,46)$. In turn, ROS were proposed as primary redox-signaling molecules driving OPCs toward either differentiation or self-renewal (47). Neural progenitor cells exposed to $\mathrm{H}_{2} \mathrm{O}_{2}$ in the 50-100 $\mu \mathrm{M}$ range induced their differentiation into OL lineage cells with acceleration of their maturation (48), and progenitors with high levels of ROS differentiated better than those progenitors with low levels of ROS (49). Alteration of the intracellular redox state in OPCs by $\mathrm{N}$-acetyl-L-cysteine enhanced the proliferation of the OPCs that were already committed to differentiation (50). Theoretically, if mito- 

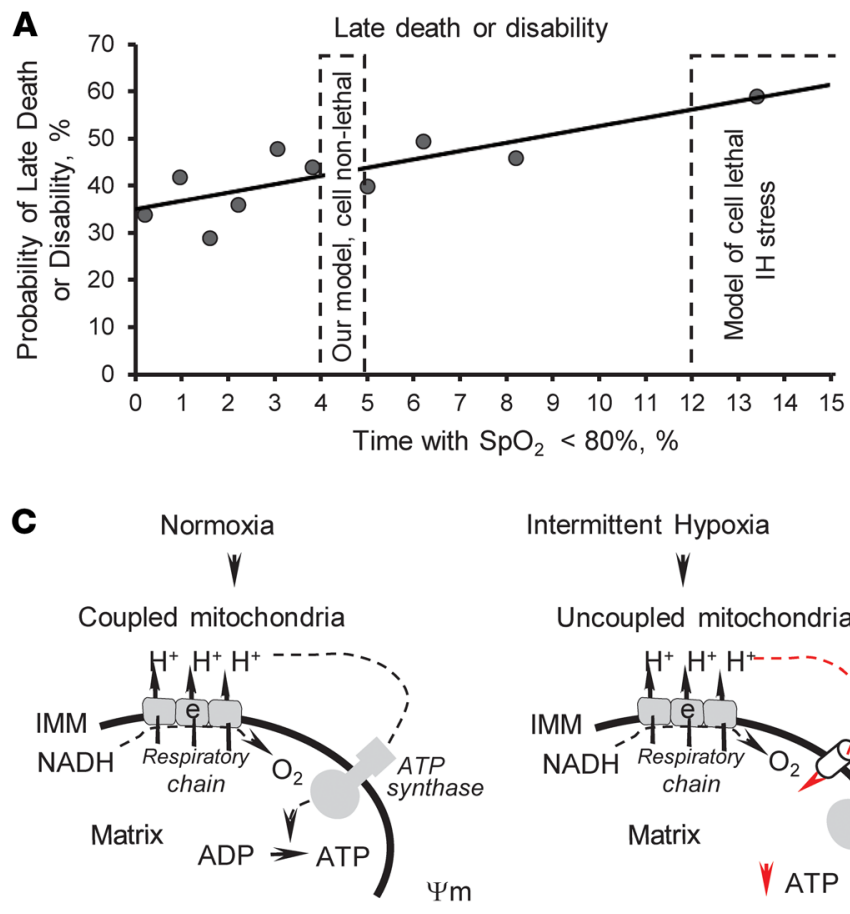

Intermittent Hypoxia

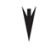

Uncoupled mitochondria

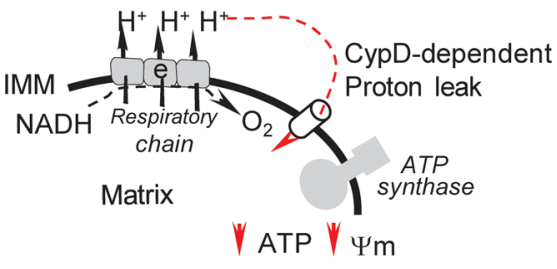

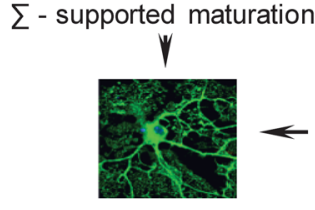

OL
$\Sigma$ - unsupported maturation

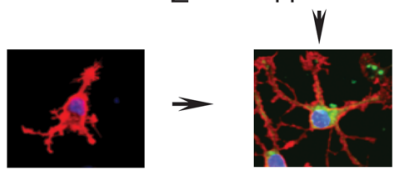

Pre-OL

WMI, Diffuse

hypomyelination
B

Normoxia

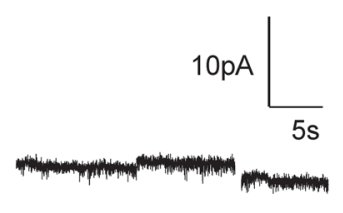

$+40 \mathrm{mV} \quad+50 \mathrm{mV}+30 \mathrm{mV}$
Intermittent Hypoxia

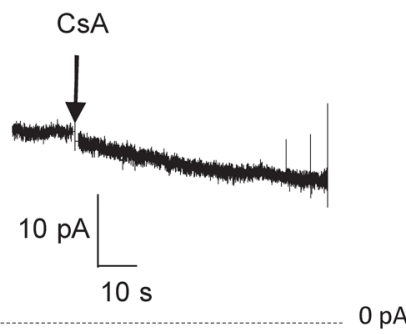

$+40 \mathrm{mV}$
Figure 10. Proposed mechanism of WMI induced by nonlethal IH stress. (A) The potential relevance of the reported (cell-lethal) paradigms of IH (27-29) inducing WMI and the used (cell-nonlethal) IH model of diffuse WMI to the outcomes and severity (the length of hypoxemia, \% of time spent with $\mathrm{SpO}_{2}<$ $85 \%$, a day) of $\mathrm{IH}$ in human premature infants (adapted from Poets et al., ref. 15). (B) Representative ion conductance recorded in mitochondria isolated from the cortex in normoxic mice and the mice subjected to sublethal IH. (C) Schematic illustration of the proposed mechanism for $\mathrm{OL}$ maturation failure and diffuse hypomyelination driven by mild, sublethal $\mathrm{IH}$ stress in premature infants. $\Sigma$, energy. chondrial ROS produced in OPCs, adjacent neurons, and glia are important for the redox-signaling for OPC differentiation, then uncoupled mitochondria may generate an ROS signal that is not sufficient for normal maturation. Testing of this hypothesis also requires a separate study.

Another potential mechanistic link between IH-driven mitochondrial ion leak, uncoupling, and arrested OL maturation relates to mitochondria-regulated $\mathrm{Ca}^{2+}$ oscillations in OPCs and pre-OLs. Mitochondrial regulation of $\mathrm{Ca}^{2+}$ oscillation has been proposed to be an important intracellular signal for OL differentiation (26, 51-53). Mitochondrial uncoupling negatively affects mitochondrial $\mathrm{Ca}^{2+}$ buffering capacity and modulates $\mathrm{Ca}^{2+}$ oscillation and $\mathrm{Ca}^{2+}$ wave propagation in differentiating OLs $(51,54)$. Thus, theoretically, arrested OPC differentiation in our model could also be mediated by dysregulated $\mathrm{Ca}^{2+}$ oscillation due to excessive mitochondrial ion leak.

In conclusion, our study offers experimental evidence supporting a potentially novel, clinically relevant mechanism contributing to OL maturational failure and diffuse hypomyelination seen in WMI in premature infants. Given that sublethal chronic IH stress is the most common manifestation of cardiorespiratory and cerebral immaturity in VLBW infants, we propose the following mechanistic sequence of events: IH, CypD-dependent mitochondrial proton leak, uncoupled mitochondrial respiration, nonlethal energy deficit, maturational failure of OLs, and deficient myelin- ation (Figure 10C). Because of the absence of excessive cerebral cellular death in the model, our study dissected cell death-driven mechanisms of WMI and introduced a potentially novel mechanism explaining the developmental failure of surviving OLs. We did not directly explore mitochondrial function in other cells (neurons, astrocytes) known to play a signaling role in the maturation of OPCs and myelination. Therefore, the mechanism proposed here is limited to the differentiating OPCs in the setting of cell-nonlethal IH stress. Further studies should assess the impact of nonlethal mitochondrial dysfunction in neurons to OLs' dysmaturation and arrested myelination. To date, however, this work offers strong evidence in support of OL precursors' specific nonlethal mitochondrial dysfunction via CypD-sensitive mitochondrial proton leak as the mechanism contributing to diffuse WMI in premature infants experiencing chronic IH stress.

\section{Methods}

\section{The model of diffuse WMI and study groups}

As we described (16), C57BL/6J newborn mice were exposed to $30 \mathrm{IH}$ events a day from P1 until P14. Each event consisted of 3 minutes of hypoxia followed by 5 minutes of reoxygenation in room air. Hypoxemia was induced by the exposure to $8 \% \mathrm{O}_{2} / 92 \% \mathrm{~N}_{2}$ at the ambient temperature of $34^{\circ} \mathrm{C}$. To obtain the evidence for a mechanistic role of mitochondrial uncoupling in the pathogenesis of WMI, a separate 
group of normoxic mice was exposed to the mitochondrial uncoupler 2,4 DNP (MilliporeSigma). DNP (5 $\mu \mathrm{g} / \mathrm{g}$ ) or vehicle (10\% DMSO in normal saline, $10 \mu \mathrm{L} / \mathrm{g}$ ) was i.p. injected daily for the initial 3 days and every other day until 14 days of life, as we described (55).

To dissect the role of CypD in IH-induced mitochondrial proton leak and uncoupling, mice born to CypD heterozygotes $\left(\mathrm{CypD}^{+/-}\right)$ were exposed to the same IH paradigm, followed by their genotyping at the end of the experiment. Littermates separated from the dams kept in room temperature air at $34^{\circ} \mathrm{C}$ served as normoxic controls. CypD $^{-/-}$mice (B6;129-Ppif ${ }^{\text {tm1Mar; }}$ CypD-KO), stock 022308, were purchased from The Jackson Laboratory and bred in the animal facility at Columbia University. Starting at P10 and upon completion of IH or DNP exposure, all mice were tested for their sensorimotor performance followed by an assessment of OL maturation and myelination. In the experiment with $\mathrm{CypD}^{-/-}$mice and their WT littermates, the extent of axonal myelination was also examined in adulthood at P80P90. Changes in $\mathrm{SpO}_{2}$ during $\mathrm{IH}$ episodes were recorded in a separate cohort of WT and $\mathrm{CypD}^{-/-} \mathrm{P} 8$ mice using a mouse vital sign monitor (Starr Life Sciences), as we described (16).

In addition to the genetic alteration of $\mathrm{CypD}$, in a separate cohort of mice, we used pharmacological inhibition of CypD with CsA (5 $\mu \mathrm{g} / 10 \mu \mathrm{L} / \mathrm{g}$ of the body weight, i.p.) and compared their outcomes with normoxic and hypoxic littermates treated with the vehicle (10 $\mu \mathrm{L} / \mathrm{g}, 10 \%$ DMSO, i.p.). The CsA was administered every other day starting at P2. All study groups and primary experimental readouts are depicted in Figure 1A.

\section{Assessment of sensorimotor performance}

All 4 tests were performed as we described (16).

Olfactory discrimination test. An olfactory discrimination test adapted for the P10 mice was performed as we described. In the standard plastic mouse cage, one lateral zone was filled with the home care bedding and the opposite zone with freshly made bedding. A pup was placed in the center (neutral zone) at a distance equal to the length of the pup's body. The time required for the animal to walk into the home bedding zone was recorded. The allotted time to enter the home bedding zone was 60 seconds. If a mouse failed to enter the home bedding zone, a maximum time of 60 seconds was assigned. Each pup was given 2 trials.

The balance beam test. Upon completion of $\mathrm{IH}$, a P15 mouse was placed on one end of the beam (length $80 \mathrm{~cm}$, diameter $[\varnothing] 1.3 \mathrm{~cm}$ ) and the time required to cross the beam was recorded. Each animal was given 1 training trial and 2 trials 10 minutes apart.

Wire-holding test. Mice (P15) were suspended on a $30 \mathrm{~cm}$ wire $(\varnothing 0.5$ $\mathrm{mm}$ ) by the forepaws. The time (seconds) that the mouse was able to hold onto the wire before falling was recorded. Each mouse was given 2 trials.

Walking initiation test. The walking initiation test was performed as previously described. To assess locomotion and extinguishing of pivoting behavior (56), a P10 mouse was placed in the center of a 13 $\mathrm{cm} \varnothing$ circle and the time (seconds) required to exit the circle with both forelimbs outside was recorded. In all tests, the mean value of the 2 trials was used for analysis.

\section{Assessment of cerebral myelination and OL maturation}

After completion of neurofunctional testing, P16 mice were euthanized. An entire single hemisphere was used for quantitation of MBP and CNP-ase using Western blot analysis (13). In brief, cere- bral MBP and CNP-ase levels were detected with MBP-Ab (US Biological, M9758, 1:1000) and monoclonal anti-CNP-ase Ab (MilliporeSigma, C-5822, 1:10,000) and the content was expressed as a density ratio between the protein-specific and $\beta$-actin bands. Images were captured using FluorChem M system (Protein Simple). Randomly selected mice were used for immunohistochemical analysis of OL maturational state. In brief, under isoflurane anesthesia, mice were intracardially perfused, and their brains were fixed in $4 \%$ paraformaldehyde in $0.1 \mathrm{M}$ phosphate buffer, coronally sectioned $(50 \mu \mathrm{m})$, and used for detection and quantitation of mature OLs defined as $\mathrm{CC}^{+}$and Olig2 ${ }^{+}$cells and OPCs ( $\mathrm{NG}^{+}$cells). We used a mouse monoclonal anti-APC (CC1) Ab (MilliporeSigma, MABC200, 1:400) and a mouse monoclonal anti-Olig2 Ab (R\&D Systems, Bio-Techne, AF2418, 1:500) and anti-NG2 (MilliporeSig$\mathrm{ma}, \mathrm{AB} 5320,1: 200)$. Olig2 ${ }^{+}$and $\mathrm{CC}^{+}$were considered mature OLs and quantified as a percentage of all Olig $2^{+}$cells. Cells were counted in a frame, $100 \times 400$ pixels $(62 \times 251 \mu \mathrm{m})$, applied to the corpus callosum and external capsule in all images. Six different images (2 images per each of 3 coronal sections at the bregma levels, $0 \mathrm{~mm}$, $+0.5 \mathrm{~mm}$, and $+1 \mathrm{~mm}$ ) of the external capsule and corpus callosum were analyzed for each mouse using confocal microscopy (Nikon, A1R MP+). Images were captured at the resolution of $1024 \times 1024$ pixels under original magnification $\times 20$ (Z-stack 9 sections, $1 \mu \mathrm{m})$, with a frame of $630 \times 630 \mu \mathrm{m}$.

The extent of cellular degeneration was examined using TUNEL assay (DeadEnd Fluorometric TUNEL system, Promega, G3250) and by immunostaining the brains for cleaved caspase-3-positive cells (Asp175 Ab, Cell Signaling Technology, 9661) in P5 and P9-10 mice exposed to IH and compared with that in normoxic controls. Positive cells were examined under original magnification $\times 20$, and quantified in the fields of the cortex and external capsule containing no less than 2 positive cells. Three fields per mouse were analyzed and cells were counted and expressed as the percentage of all Dapi ${ }^{+}$cells per field.

\section{Electron microscopy}

Randomly selected mice from each experimental group were anesthetized with isoflurane and perfused intracardially with $1 \%$ glutaraldehyde and $4 \%$ paraformaldehyde in $0.1 \mathrm{M}$ sodium phosphate buffer, $\mathrm{pH}$ 7.4. Brains were removed, fixed, postfixed in $\mathrm{OsO}_{4}$, and embedded in Epon. Ultrathin transverse sections of corpus callosum were stained with uranyl acetate and lead citrate and examined under a JEOL 100 S electron microscope. Images were captured with a Hamamatsu ORCA HR camera. Axonal density was estimated as described (57) and expressed as a number of axons per $100 \mu \mathrm{m}^{2}$. The extent of axonal myelination was "blindly" quantified by the calculation of g-ratio (axonal diameter divided by the diameter of axon + myelin), as we described (16). The axonal caliber and myelinated axonal diameter were measured using Image $(\mathrm{NIH})$. Axons with diameters less than $300 \mathrm{~nm}$ were not analyzed. At least 4 images per animal captured at original magnification $\times 20,000$ were used. A minimum of 50 axons per animal were analyzed, and mean value was used for statistical analysis.

\section{In vitro studies}

Primary OPC (NG2 cell) cultures were prepared as previously described (58). Briefly, meninges-free cortices from neonatal mice (P1) were dissected, digested with papain, and gently forced through 
a $40 \mu \mathrm{m}$ Corning mesh to form a single-cell suspension. After centrifugation, pellets were resuspended in DMEM containing 10\% FBS, GlutaMax (1×), and penicillin/streptomycin and plated in poly-D-lysine-coated flasks $(0.1 \mathrm{mg} / \mathrm{mL})$. After 9 days of incubation, flasks were shaken using a horizontal orbital shaker $(200 \mathrm{rpm})$ at $37^{\circ} \mathrm{C}$ for 16 to 18 hours. Medium with free-floating cells was collected, centrifuged, and pellets were resuspended in F12/DMEM containing GlutaMax (1×), 0.01\% BSA, 100 units $/ \mathrm{mL}$ penicillin, $100 \mu \mathrm{g} / \mathrm{mL}$ streptomycin, N2 and B27 supplements, and growth factors $(20 \mathrm{ng} / \mathrm{mL}$ PDGF-AA, 20 $\mathrm{ng} / \mathrm{mL}$ FGF). Cells were plated into poly-D-lysine- and laminin-coated Nunc 8-well glass slides $\left(2 \times 10^{4}\right.$ cells/well $)$ and incubated for 48 hours. To induce differentiation, growth factors in the medium were substituted by ciliary neurotrophic factor $(50 \mathrm{ng} / \mathrm{mL})$ and $\mathrm{T}_{3}(0.4 \mu \mathrm{g} /$ $\mathrm{mL})$. Prior to induction of differentiation, cells were examined for expression of Olig2 (R\&D Systems, Bio-Techne, AF2418, 1:500) and NG2 (MilliporeSigma, AB5320, 1:200) markers.

IH paradigm or pharmacological mitochondrial uncoupling in vitro were started simultaneously with the induction of differentiation of primary OPCs. IH was carried out using differentiation medium prebubbled with $\mathrm{N}_{2}(95 \%) / \mathrm{CO}_{2}(5 \%)$, followed by incubation in a hypoxic environment $\left(<0.2 \% \mathrm{O}_{2}, 5 \% \mathrm{CO}_{2}, 37^{\circ} \mathrm{C}\right)$ for 30 minutes cycled by 30 minutes of reoxygenation in room temperature air. These IH cycles were repeated 5 times daily for 5 days. Cells cultured in the normoxic environment with the same protocol of medium exchange were controls. To uncouple mitochondria, the normoxic normoxic medium was supplemented with $200 \mu \mathrm{M}$ of DNP. Every 30 minutes, DNP-containing medium was substituted by DNP-free medium for 30 minutes, 5 times a day for 5 days. PBS was used as a vehicle. The extent of OPC differentiation was examined at the end of the second and fifth days of differentiation by immunostaining for NG2 and CNP-ase (MilliporeSigma, C5922, 1:100) and NG2 and MBP (MilliporeSigma, AB5320, 1:100).

\section{Assessment of mitochondrial respiration}

Subcortical nonsynaptosomal mitochondria were isolated using a Percoll gradient and differential centrifugation as described (59) from P5 mice exposed to IH for 4 days at the end of the 15 th episode on day 5 of exposure. Mitochondrial respiration was measured using a Clark-type electrode (Hansatech). Mitochondria ( $0.05 \mathrm{mg}$ of protein) were added to $0.5 \mathrm{~mL}$ of respiration buffer: $10 \mathrm{mM}$ MOPS-Tris, $\mathrm{pH} 7.4,120 \mathrm{mM}$ $\mathrm{KCl}, \mathrm{KH}_{2} \mathrm{PO}_{4} 1 \mathrm{mM}$, EGTA $10 \mu \mathrm{M}, 0.2 \mathrm{mg} / \mathrm{mL}$ of BSA, $30 \mu \mathrm{M}$ Ap5A [P1,P5-di(adenosine 5')-pentaphosphate], $10 \mathrm{mM}$ glutamate, and 5 $\mathrm{mM}$ malate at $32^{\circ} \mathrm{C}$. Phosphorylating respiration (state 3 ) was initiated by adding $100 \mathrm{nmol}$ of ADP (MilliporeSigma). Rates of $\mathrm{O}_{2}$ consumption were expressed in $\mathrm{nmol}_{2} / \mathrm{mg}$ mitochondrial protein $/ \mathrm{min}$. The respiratory control ratio was calculated as the ratio of the state 3 respiration rate to the resting respiration rate (state 4) recorded after the phosphorylation of ADP was completed. To control for any substrate transport-linked inhibition of respiratory chain, DNP (35 nM) was used to initiate uncoupled respiration. In each experiment, the ADP/O ratio (nanomoles of ADP phosphorylated to ATP during consumption of 1 atom of $\mathrm{O}_{2}$ ) was calculated.

Assessment of $\Delta \Psi_{\mathrm{m}}$ was carried out using FACS analysis (CantoII flow cytometer). Differentiating OPCs (day 2 of differentiation) were incubated with TMRE ( $20 \mathrm{nM}, 15$ minutes). Cells were harvested using trypLE solution (Thermo Fisher Scientific), centrifuged, and the pellet was suspended in PBS. To control for nonspecific fluorescence, after measurement of $\Psi \mathrm{m}$-associated fluorescence, each sample was incubated with $20 \mu \mathrm{M}$ of FCCP (MilliporeSigma) for 5 minutes, and the fluorescence was measured again. The value of the fluorescence obtained after FCCP treatment was subtracted from the initial value, and the $\Psi \mathrm{m}$-defining fluorescence was expressed as the percentage of the normoxic controls for each experiment.

Mitochondrial $\mathrm{Ca}^{2+}$ accumulation was determined as we described (60). Briefly, mitochondria were isolated from subcortical brains without EGTA and $0.1 \mathrm{mg} / \mathrm{mL}$ incubated in $10 \mathrm{mM}$ Tris-MOPS buffer $(\mathrm{pH}$ 7.4) containing $120 \mathrm{mM} \mathrm{KCl}, 1 \mathrm{mM} \mathrm{K}_{2} \mathrm{HPO}_{4}, 10 \mu \mathrm{M}$ EGTA, $5 \mathrm{mM}$ sodium succinate, $2.5 \mathrm{mM}$ sodium glutamate, and $1 \mu \mathrm{M} 5 \mathrm{~N}$-Calcium Green. After the stabilization of fluorescence, alamethicin $(50 \mu \mathrm{g} / \mathrm{mL})$ was added to release $\mathrm{Ca}^{2+}$. Changes in calcium fluorescence were recorded. At 200 seconds of recording, calcium fluorescence of normoxic and IH samples was normalized by subtracting a nonspecific alamethicin-induced fluorescence and quantified in fluorescence units.

Measurement of phosphorylated AMP-activated protein kinase was performed immediately after $10 \mathrm{IH}$ events by Western blot, using phospho-AMPK- $\alpha$ (Thr172) rabbit mAb, dilution 1:5000, applied overnight at $4^{\circ} \mathrm{C}$ (Cell Signaling Technology, 2535). All samples were also probed for the total AMPK- $\alpha$ content (Cell Signaling Technology, 2532), and no differences between groups were detected.

Assessment of cerebral ATP content was done using a bioluminescent assay kit CLS II (Roche, 11699695001). For the ATP assay, mice were euthanized immediately after $10 \mathrm{IH}$ exposures. Samples were immediately frozen in liquid nitrogen. Determination of ATP and preparation of standard curve were performed according to the manufacturer's protocol, and 5\% TCA was used for the ATP extraction from the brain homogenate.

\section{Measurement of mitochondrial transmembrane electrical current (patch-clamp)}

Mitochondria were isolated from pre-OLs at the end of differentiation day 2 and placed into the standard $150 \mathrm{mM} \mathrm{KCl}$ buffer $(\mathrm{pH}=7.4)$ containing no substrate. In this deenergized condition, mitochondria swelled into mitoplasts. Membrane patches were excised from mitoplasts after formation of a giga-seal using micropipettes with resistances of 20 to $40 \mathrm{M} \Omega$ at room temperature (61). The solution for patch-clamp recordings was symmetrical, $150 \mathrm{mM} \mathrm{KCl,} 5 \mathrm{mM}$ HEPES, $\mathrm{pH}$ 7.4. The voltage clamp was performed with the excised configuration of the patch-clamp technique using 1 channel amplifier eONE (Elements SRL) in the inside-out mode. Voltages are reported as pipette potentials. CsA (MilliporeSigma) at the final concentration of $2 \mu \mathrm{M}$ was used for the inhibition of the channel activity and was delivered using a microperfusion system. Clampfit 10.7 (Molecular Devices) and Origin 2018 (OriginLab Corporation) were used for analysis of channel activity and statistical analysis.

\section{Statistics}

All data are shown as mean \pm SEM. Statistical analysis details are stated in the figure legends. Differences between 2 groups were assessed by unpaired, 1-tailed $t$ test or nonparametric Mann-Whitney $U$ test. Differences between a control group and 2 or more other groups were analyzed using 1-way ANOVA with Dunnett's multiple-comparisons post hoc test or Kruskal-Wallis test with Dunn's multiple-comparisons post hoc test. Two-way ANOVA was used to compare multiple groups considering 2 independent variables. Differences between multiple 
groups were analyzed by post hoc Tukey's multiple-comparisons test with the FDR-adjusted $P$ values. Data differences were considered significant if the $P$ value was less than or equal to 0.05 .

\section{Study approval}

All experiments were approved by the Columbia University Institutional Animal Care and Use Committee in accordance with Association for Assessment and Accreditation of Laboratory Animal Care guidelines under protocol AABA5474.

\section{Author contributions}

$\mathrm{ZN}$ and VT generated the idea, designed and conducted experiments, analyzed data, and wrote the manuscript. JG and AG participated in the data analysis and manuscript writing and editing. AS and VT conducted statistical analysis, SS conducted in vivo experiments, and MN and EP conducted patch-clamp experiments, analyzed data, and participated in manuscript writing and editing.

\section{Acknowledgments}

This work was supported by NIH grant NS 099109 and partially supported by grants NS 100850 (VT) and NS 112381 (AG). Image processing for this work was performed in the Confocal and Specialized Microscopy Shared Resource of the Herbert Irving Comprehensive Cancer Center at Columbia University, supported by NIH grant P30 CA013696 (National Cancer Institute).

Address correspondence to: Vadim S. Ten, 650 West 168 Street, BB-4407, New York, New York 10032, USA. Email: vt82@columbia.edu.
1. Hamilton BE, Miniño AM, Martin JA, Kochanek KD, Strobino DM, Guyer B. Annual summary of vital statistics: 2005. Pediatrics. 2007;119(2):345-360.

2. Volpe JJ. Cerebral white matter injury of the premature infant-more common than you think. Pediatrics. 2003;112(1 pt 1):176-180.

3. Dyet LE, et al. Natural history of brain lesions in extremely preterm infants studied with serial magnetic resonance imaging from birth and neurodevelopmental assessment. Pediatrics. 2006;118(2):536-548.

4. Duncan AF, Bann CM, Dempsey A, Peralta-Carcelen M, Hintz S, Eunice Kennedy Shriver National Institute of Child Health Development Neonatal Research Network. Behavioral deficits at 18-22 months of age are associated with early cerebellar injury and cognitive and language performance in children born extremely preterm. JPediatr. 2019;204:148-156.e4.

5. Back SA. White matter injury in the preterm infant: pathology and mechanisms. Acta Neuropathol. 2017;134(3):331-349.

6. Volpe JJ, Kinney HC, Jensen FE, Rosenberg PA. The developing oligodendrocyte: key cellular target in brain injury in the premature infant. Int J Dev Neurosci. 2011;29(4):423-440.

7. Adams-Chapman I, et al. Neurodevelopmental impairment among extremely preterm infants in the Neonatal Research Network. Pediatrics. 2018;141(5):e20173091.

8. Buser JR, et al. Arrested preoligodendrocyte maturation contributes to myelination failure in premature infants. Ann Neurol. 2012;71(1):93-109.

9. Harris JJ, Attwell D. The energetics of CNS white matter. J Neurosci. 2012;32(1):356-371.

10. Dobbing J, Sands J. Comparative aspects of the brain growth spurt. Early Hum Dev. 1979;3(1):79-83.

11. Erecinska M, Cherian S, Silver IA. Energy metabolism in mammalian brain during development. Prog Neurobiol. 2004;73(6):397-445.

12. Bates TE, Almeida A, Heales SJ, Clark JB. Postnatal development of the complexes of the electron transport chain in isolated rat brain mitochondria. Dev Neurosci. 1994;16(5-6):321-327.

13. Amaral AI, Hadera MG, Tavares JM, Kotter MR, Sonnewald U. Characterization of glucose-related metabolic pathways in differentiated rat oligodendrocyte lineage cells. Glia. 2016;64(1):21-34.

14. Martin RJ, Wang K, Köroğlu O, Di Fiore J, Kc P.
Intermittent hypoxic episodes in preterm infants: do they matter? Neonatology. 2011;100(3):303-310.

15. Poets CF, et al. Association between intermittent hypoxemia or bradycardia and late death or disability in extremely preterm infants. JAMA. 2015;314(6):595-603.

16. Juliano C, et al. Mild intermittent hypoxemia in neonatal mice causes permanent neurofunctional deficit and white matter hypomyelination. Exp Neurol. 2015;264:33-42.

17. Kariman K, Chance B, Burkhart DS, Bolinger LA. Uncoupling effects of 2,4-dinitrophenol on electron transfer reactions and cell bioenergetics in rat brain in situ. Brain Res. 1986;366(1-2):300-306.

18. Ichas F, Jouaville LS, Mazat JP. Mitochondria are excitable organelles capable of generating and conveying electrical and calcium signals. Cell. 1997;89(7):1145-1153.

19. Lemasters JJ, Theruvath TP, Zhong Z, Nieminen AL. Mitochondrial calcium and the permeability transition in cell death. Biochim Biophys Acta. 2009;1787(11):1395-1401.

20. Ortinau C, Neil J. The neuroanatomy of prematurity: normal brain development and the impact of preterm birth. Clin Anat. 2015;28(2):168-183.

21 . Volpe JJ. Brain injury in premature infants: a complex amalgam of destructive and developmental disturbances. Lancet Neurol. 2009;8(1):110-124.

22. Dean JM, et al. Strain-specific differences in perinatal rodent oligodendrocyte lineage progression and its correlation with human. Dev Neurosci. 2011;33(3-4):251-260.

23. Craig A, et al. Quantitative analysis of perinatal rodent oligodendrocyte lineage progression and its correlation with human. Exp Neurol. 2003;181(2):231-240.

24. Dryer SE, Dryer RL, Autor AP. Enhancement of mitochondrial, cyanide-resistant superoxide dismutase in the livers of rats treated with 2,4-dinitrophenol. J Biol Chem. 1980;255(3):1054-1057.

25. Buffa P, Carafoli E, Muscatello U. Mitochondrial biochemical lesion and pyrogenic effect of pentachlorophenol. Biochem Pharmacol. 1963;12:769-778.

26. Bonora M, et al. Tumor necrosis factor- $\alpha$ impairs oligodendroglial differentiation through a mitochondria-dependent process. Cell Death Differ. 2014;21(8):1198-1208.

27. Douglas RM, et al. Neuronal death during com- bined intermittent hypoxia/hypercapnia is due to mitochondrial dysfunction. Am J Physiol, Cell Physiol. 2010;298(6):C1594-C1602.

28. Cai J, et al. Mouse intermittent hypoxia mimicking apnoea of prematurity: effects on myelinogenesis and axonal maturation. J Pathol. 2012;226(3):495-508.

29. Oorschot DE, et al. Spectrum of short- and longterm brain pathology and long-term behavioral deficits in male repeated hypoxic rats closely resembling human extreme prematurity. J Neurosci. 2013;33(29):11863-11877.

30. Di Fiore JM, MacFarlane PM, Martin RJ. Intermittent hypoxemia in preterm infants. Clin Perinatol. 2019;46(3):553-565.

31. Nakagawa T, et al. Cyclophilin D-dependent mitochondrial permeability transition regulates some necrotic but not apoptotic cell death. Nature. 2005;434(7033):652-658.

32. Ichas F, Mazat JP. From calcium signaling to cell death: two conformations for the mitochondrial permeability transition pore. Switching from lowto high-conductance state. Biochim Biophys Acta. 1998;1366(1-2):33-50.

33. Korge P, Yang L, Yang JH, Wang Y, Qu Z, Weiss $\mathrm{JN}$. Protective role of transient pore openings in calcium handling by cardiac mitochondria. J Biol Chem. 2011;286(40):34851-34857.

34. Herzig S, Shaw RJ. AMPK: guardian of metabolism and mitochondrial homeostasis. Nat Rev Mol Cell Biol. 2018;19(2):121-135.

35. Bizzozero OA, Sanchez P, Tetzloff SU. Effect of ATP depletion on the palmitoylation of myelin proteolipid protein in young and adult rats. $J \mathrm{Neu}$ rochem. 1999;72(6):2610-2616.

36. Fünfschilling U, et al. Glycolytic oligodendrocytes maintain myelin and long-term axonal integrity. Nature. 2012;485(7399):517-521.

37. Rao VTS, et al. Distinct age and differentiation-state dependent metabolic profiles of oligodendrocytes under optimal and stress conditions. PLoS One. 2017;12(8):e0182372.

38. Schoenfeld R, et al. Oligodendroglial differentiation induces mitochondrial genes and inhibition of mitochondrial function represses oligodendroglial differentiation. Mitochondrion. 2010;10(2):143-150.

39. Ziabreva I, et al. Injury and differentiation following inhibition of mitochondrial respiratory chain complex IV in rat oligodendrocytes. Glia. 
2010;58(15):1827-1837.

40. Wang Y, et al. Hyperforin promotes mitochondrial function and development of oligodendrocytes. J Neurochem. 2011;119(3):555-568.

41. Keller D, Erö C, Markram H. Cell densities in the mouse brain: a systematic review. Front Neuroanat. 2018;12:83.

42. Stevens B, Porta S, Haak LL, Gallo V, Fields RD. Adenosine: a neuron-glial transmitter promoting myelination in the CNS in response to action potentials. Neuron. 2002;36(5):855-868.

43. Fields RD. Imaging single photons and intrinsic optical signals for studies of vesicular and non-vesicular ATP release from axons. Front Neuroanat. 2011;5:32.

44. Murphy MP. How mitochondria produce reactive oxygen species. Biochem J. 2009;417(1):1-13.

45. Korshunov SS, Skulachev VP, Starkov AA. High protonic potential actuates a mechanism of production of reactive oxygen species in mitochondria. FEBS Lett. 1997;416(1):15-18.

46. Starkov AA, Fiskum G. Regulation of brain mitochondrial $\mathrm{H} 2 \mathrm{O} 2$ production by membrane potential and $\mathrm{NAD}(\mathrm{P}) \mathrm{H}$ redox state. J Neurochem. 2003;86(5):1101-1107.

47. Olguín-Albuerne M, Morán J. Redox signaling mechanisms in nervous system development. Antioxid Redox Signal. 2018;28(18):1603-1625.

48. Pérez Estrada C, Covacu R, Sankavaram SR, Svensson M, Brundin L. Oxidative stress increases neurogenesis and oligodendrogenesis in adult neural progenitor cells. Stem Cells Dev. 2014;23(19):2311-2327.

49. Smith J, Ladi E, Mayer-Proschel M, Noble M. Redox state is a central modulator of the balance between self-renewal and differentiation in a dividing glial precursor cell. Proc Natl Acad Sci U S A. 2000;97(18):10032-10037.

50. Noble M, Smith J, Power J, Mayer-Pröschel M. Redox state as a central modulator of precursor cell function. Ann N Y Acad Sci. 2003;991:251-271.

51. De Nuccio C, et al. Peroxisome proliferator-activated receptor $\gamma$ agonists accelerate oligodendrocyte maturation and influence mitochondrial functions and oscillatory $\mathrm{Ca}(2+)$ waves. J Neuropathol Exp Neurol. 2011;70(10):900-912.

52. Bernardo A, De Simone R, De Nuccio C, Visentin $\mathrm{S}$, Minghetti L. The nuclear receptor peroxisome proliferator-activated receptor- $\gamma$ promotes oligodendrocyte differentiation through mechanisms involving mitochondria and oscillatory $\mathrm{Ca} 2+$ waves. Biol Chem. 2013;394(12):1607-1614.

53. Simpson PB, Russell JT. Mitochondrial Ca2+ uptake and release influence metabotropic and ionotropic cytosolic $\mathrm{Ca} 2+$ responses in rat oligodendrocyte progenitors. J Physiol (Lond). 1998;508(pt 2):413-426.

54. Gunter TE, Gunter KK, Sheu SS, Gavin CE. Mitochondrial calcium transport: physiological and pathological relevance. Am J Physiol. 1994;267(2 pt 1):C313-C339.

55. Ratner V, Sosunov SA, Niatsetskaya ZV, Utki-
na-Sosunova IV, Ten VS. Mechanical ventilation causes pulmonary mitochondrial dysfunction and delayed alveolarization in neonatal mice. Am J Respir Cell Mol Biol. 2013;49(6):943-950.

56. Hill JM, Lim MA, Stone MM. Developmental milestones in the newborn mouse. In: Gozes I, ed. Neuropeptide Techniques. Humana Press; 2008:131-149. Neuromethods; vol. 39.

57. LaMantia AS, Rakic P. Axon overproduction and elimination in the corpus callosum of the developing rhesus monkey. J Neurosci. 1990;10(7):2156-2175.

58. O'Meara RW, Ryan SD, Colognato H, Kothary R. Derivation of enriched oligodendrocyte cultures and oligodendrocyte/neuron myelinating co-cultures from post-natal murine tissues. J Vis Exp. 2011;(54):3324.

59. Niatsetskaya ZV, et al. The oxygen free radicals originating from mitochondrial complex I contribute to oxidative brain injury following hypoxia-ischemia in neonatal mice. J Neurosci. 2012;32(9):3235-3244.

60. Utkina-Sosunova IV, Niatsetskaya ZV, Sosunov SA, Ratner VI, Matsiukevich D, Ten VS. Nelfinavir inhibits intra-mitochondrial calcium influx and protects brain against hypoxic-ischemic injury in neonatal mice. PLoS One. 2013;8(4):e62448.

61. Neginskaya MA, et al. ATP synthase c-subunit-deficient mitochondria have a small cyclosporine A-sensitive channel, but lack the permeability transition pore. Cell Rep. 2019;26(1):11-17.e2. 